\title{
A common East Asian-specific ALDH2 mutation causes obesity and insulin resistance: therapeutic effect of reducing toxic aldehydes by ALDH2 activation
}

\section{Yi-Cheng Chang ( $\sim$ b83401040@gmail.com )}

College of Medicine, National Taiwan University https://orcid.org/0000-0002-8077-5011

\section{Hsiao-Lin Lee}

College of Medicine, National Taiwan University

\section{Wenjin Yang}

Foresee Pharmaceuticals, Co. Ltd

\section{Meng-Lun Hsieh}

College of Medicine, National Taiwan University

Cai-Cin Liu

College of Medicine, National Taiwan University

\section{Tung-Yuan Lee}

College of Medicine, National Taiwan University

\section{Jing-Yong Huang}

College of Medicine, National Taiwan University

\section{Jiun-Yi Nong}

College of Medicine, National Taiwan University

\section{Fu-An Li}

Institute of Biomedical Sciences, Academia Sinica https://orcid.org/0000-0002-0580-7765

\section{Hsiao-Li Chuang}

National Laboratory Animal Center, National Applied Research Laboratories

\section{Zhi-Zhong Ding}

College of Medicine, National Taiwan University

\section{Wei-Lun Su}

College of Medicine, National Taiwan University

\section{Li-Yun Chueh}

College of Medicine, National Taiwan University

\section{Yi-Ting Tsai}

Laboratory Animal Center, College of Medicine, National Taiwan University

\section{Che-Hong Chen}

Department of Chemical and Systems Biology, Stanford University School of Medicine 


\section{Daria Mochly-Rosen}

Stanford University School of Medicine https://orcid.org/0000-0002-6691-8733

\section{Lee-Ming Chuang}

National Taiwan University Hospital

\section{Article}

Keywords: Acetaldehyde Metabolizing Enzyme, Missense Mutation, Homozygous Knock-in Mice, Thermogenesis, Substrate-enzyme Collision, Glucose Homeostasis

Posted Date: December 2nd, 2020

DOl: https://doi.org/10.21203/rs.3.rs-104384/v1

License: (c) (i) This work is licensed under a Creative Commons Attribution 4.0 International License. Read Full License 


\section{Abstract}

Obesity and type 2 diabetes have reached pandemic proportion. In particular, the population with diabetes is expected to rise rapidly in East and South Asia. ALDH2 (acetaldehyde dehydrogenase 2, mitochondrial) is the key metabolizing enzyme of acetaldehyde and other toxic aldehydes, such as 4-hydroxynonenal (4$\mathrm{HNE}$.

A missense mutation, Glu504Lys of $A L D H 2$ (denoted as the $A L D H 2 * 2$ allele) is prevalent in 560 million East Asians, resulting in reduced ALDH2 enzymatic activity. We found that $A / d h 2 * 2 / 2$ homozygous knock-in (KI) mice mimicking human Glu504Lys mutation were prone to develop diet-induced obesity, glucose intolerance, insulin resistance, and fatty liver on a high-fat high-sucrose diet compared with controls. The Aldh $2 \mathrm{KI}$ mice demonstrated reduced energy expenditure and thermogenesis. Proteomic analyses of the brown adipose tissue (BAT) of the Aldh2 KI mice identified increased 4-HNE-adducted proteins involved in fatty acid oxidation and electron transport chain. Fatty acid oxidation rate and mitochondrial electron transport activity were reduced in the BAT of the Aldh $2 \mathrm{KI}$ mice, which explained the decrease in thermogenesis and energy expenditure.

AD-9308 is a water-soluble prodrug of a potent and highly selective ALDH2 activator AD-5591. In vitro, AD-5591 enhanced both WT and mutant ALDH2 enzymatic activities. AD-9308 allosterically activates ALDH2 mainly by partially blocking the substrate exit tunnel, thereby accelerating the substrate-enzyme collision. In vivo, AD-9308 treatment reduced serum 4-HNE levels, ameliorated diet-induced obesity and fatty liver, and improved glucose homeostasis in both Aldh2 WT and KI mice dose-dependently. Our data highlight the therapeutic potential of reducing toxic aldehyde levels by activating ALDH2 for treating metabolic diseases.

\section{Introduction}

Obesity and type 2 diabetes have reached pandemic levels. The 2019 International Diabetes Federation reported that there were 463 million people living with diabetes worldwide. This number is projected to increase rapidly to 578 million in $2030^{7}$. East Asia and South Asia, the most populous regions in the world, are expected to be the major contributors of new cases ${ }^{1}$. This pandemic is primarily caused by a high-calorie diet enriched in fat and sugar, sedentary lifestyle, and their interaction with genetic factors ${ }^{1}$, 2 .

Approximately $36 \%$ of East Asians (560 million) or nearly $8 \%$ of the global population, carry an inactivating missense mutation of the $A L D H 2$ gene (denoted as the $A L D H 2 \star 2$ allele), which causes Glu504Lys substitution ${ }^{3}$. This mutation results in a reduction of the ALDH2 enzymatic activity by $60-$ $80 \%$ in heterozygous carriers $\left(A L D H 2^{*} 1 /{ }^{*} 2\right)$ and $~ 90 \%$ in homozygous carriers $\left(A L D H 2 * 2 /{ }^{*} 2\right)^{4}$. $A L D H 2^{*} 2$ heterozygotes and $A L D H 2 * 2$ homozygotes exhibit sensitivity to alcohol, with presentations ranging from facial flushing, headache, and tachycardia due to a rapid increase in circulating acetaldehyde concentrations ${ }^{5,6}$. Epidemiological studies suggest a correlation between this inactivating mutation and 
several diseases including oral cancer, esophageal cancers, and blood and solid tumors associated with Fanconi anemia 7,8 . Importantly, several large-scale meta-analyses of genome-wide association studies revealed that this inactivating mutation is strongly associated with type 2 diabetes ${ }^{9}$, body mass index ${ }^{10}$, and serum lipids ${ }^{11}$ in East Asians. A validation study further confirmed a close association of $A L D H 2 \star 2$ with visceral fat distribution in 2,958 Chinese subjects $^{12}$. In addition, carriers of the inactivating $A L D H 2 * 2$ variant had 2-3 times increased risk of non-alcoholic fatty liver disease among Japanese subjects ${ }^{13}$.

The $A L D H 2$ gene encodes mitochondrial aldehyde dehydrogenase 2 (ALDH2), a major acetaldehydemetabolizing enzyme responsible for $\sim 95 \%$ acetaldehyde metabolism due to its low $\mathrm{Km}$ for acetaldehyde ${ }^{14}$. Acetaldehyde is produced by alcohol dehydrogenase (ADH) following alcohol ingestion. Acetaldehyde can also be generated endogenously from the intermediate metabolisms or by gut microbial flora ${ }^{15}$. Air pollution, thermal degradation of plastics, and spoiled food are also sources of toxic aldehyde ${ }^{16-18}$. Acetaldehyde is listed as group I carcinogen by the International Agency for Research on Cancer ${ }^{19}$. In addition to acetaldehyde, ALDH2 also metabolizes various bioactive toxic aldehydes, including acrolein, malondialdehyde and 4-hydroxynonenal (4-HNE). Among these bioactive aldehydes, the most intensively studied has been 4-HNE, a lipid peroxidation product that forms covalent adduct to macromolecules such as protein, lipid and DNA, causing cellular damage ${ }^{20-24}$.

With the advent of high-throughput screening, a small molecule, Alda-1, was identified as an activator for both the wild-type ALDH2and mutant ALDH2 enzymes ${ }^{25}$. Based on X-ray crystallography and enzyme kinetics, the binding of Alda-1 partially blocks the exit tunnel of substrates, thus increasing the likelihood of productive encounters between reaction intermediate and the catalytic site ${ }^{26}$. Administration of Alda- 1 has been effective to treat myocardial infarction, aortic aneurysm, atrial fibrillation, Alzheimer's disease and nociceptive pain in mice model by enhancing the clearance of 4-HNE and in rodent model $27,27-29$. The discovery of ALDH2 activators offers an opportunity to test the therapeutic potential of reducing toxic aldehydes for treating a variety of diseases ${ }^{30}$.

AD-9308 is a highly water soluble and orally bioavailable prodrug of a potent and highly selective ALDH2 activator AD-5591, a new generation ALDH2 activator that has the improved biological activities and pharmacological properties compared to Alda- ${ }^{31}$.

In this study, we used aldh2*2/*2 homozygous knock-in (KI) mice, which mimic the East Asian-specific Glu504Lysmutation, to evaluate the effect of this mutation on diet-induced obesity, glucose homeostasis, fatty liver, and serum lipids and tested the therapeutic effect of a novel ALDH2 activator AD-5591 by dosing its prodrug AD-9308 for treating metabolic disorders.

\section{Results}

Aldh2 KI mice carrying the East Asian-specific Glu504Lys mutation were prone to develop diet-induced obesity and fatty liver 
To assess the effect of the East Asian-specific ALDH2 Glu504Lys mutation on diet-induced obesity and related metabolic traits, $A / d h 2 \mathrm{KI}$ and WT mice littermates were placed on either regular chow or high-fat high-sucrose diet (HFHSD) for 24 weeks since the age of 4 weeks. At the end of study, the body weights of $A / d h 2 \mathrm{KI}$ and WT mice were $42.45 \pm 6.59$ and $37.78 \pm 5.54 \mathrm{~g}$ respectively (Fig. $1 \mathrm{a}, \mathrm{P}<0.0001$ ). However, no difference of body weight gain was observed between Aldh2 KI and WT mice fed a chow diet (Fig. 1b). Aldh $2 \mathrm{KI}$ mice fed on HFHSD also had more white fat, including inguinal $(1.33 \pm 0.1 \mathrm{vs} .1 .02 \pm 0.09 \mathrm{~g}, \mathrm{P}=$ $0.04)$, mesenteric $(0.68 \pm 0.091$ vs. $0.51 \pm 0.051 \mathrm{~g}, \mathrm{P}=0.077)$, and perigonadal fat $(2.29 \pm 0.27 \mathrm{vs} .1 .48 \pm$ $0.13 \mathrm{~g}, \mathrm{P}=0.004)$ and increased liver weight $(1.89 \pm 0.07 \mathrm{vs} .1 .64 \pm 0.06 \mathrm{~g}, \mathrm{P}<0.01)$, but less brown adipose tissue (BAT) weight $(0.10 \pm 0.01$ vs. $0.14 \pm 0.10 \mathrm{~g}, \mathrm{P}=0.016)$ compared with WT littermates (Fig. 1c). No such differences were found between Aldh2 KI and WT mice on chow diet except for the smaller BAT found in Aldh2 $\mathrm{KI}$ mice. Body composition analysis revealed significantly increased fat mass (12.46 \pm 0.3 vs. $8.85 \pm 1.23 \mathrm{~g}, \mathrm{P}=0.04$ ), and to a lesser extent, increased lean mass and total water conten in Aldh2 KI mice compared with the WT mice fed on HFHSD (Fig. 1d). Figure 1e showed the representative gross appearance of mice, BAT, perigonadal fat, and liver. H\&E stain using of perigonadal fat showed hypertrophic adipocytes with more crown-like necrosis in Aldh2 KI mice compared with controls (Fig. 1f, $1 \mathrm{~h}$ ). There was no difference in the number of adipocytes between the two groups (Fig. 1f-h), indicating hypertrophy rather than hyperplasia of white adipose tissue. Aldh2 KI mice also had significantly higher hepatic triglycerides contents $(0.249 \pm 0.055$ vs. $0.124 \pm 0.027 \mathrm{mg} / \mathrm{mg}$ liver tissue, $P=$ 0.005), and more severe hepatic steatosis than the WT mice on HFHSD (Fig. 1i, 1j). However, there was no difference in muscle triglycerides content (Fig. 1k). These results suggest that the East Asian-specific ALDH2 Glu504Lys mutation promoted HFHSD-induced obesity and fatty liver in mice.

\section{Aldh2 KI mice had reduced energy expenditure and impaired adaptive thermogenesis}

Energy expenditure measured by indirect calorimetry showed that $A / d h 2 \mathrm{KI}$ mice had lower energy expenditure (Fig. 2a) than WT mice. There was no difference in food intake between Aldh2 KI and WT mice (Fig, 2b).These data indicated $A / d h 2 \mathrm{KI}$ mice were prone to diet-induced obesity due to reduced energy expenditure but not intake.

Energy expenditure is composed of basal metabolic rate, physical activity, and adaptive thermogenesis including cold-induced and diet-induced thermogenesis. Indirect calorimetry showed no difference in basal metabolic rate as indicated by the energy expenditure in resting (light) phase (Fig. 2a). We also measured energy expenditure by monitoring physical activities of the mice. Daily physical activity, including wheel rotations (Fig. 2C) and travel distances (Fig. 2d) were similar between the two groups. HomeCage monitoring systems did not detect significant differences in various mouse behaviors including awakening, feeding, hanging, rearing up, resting, twisting, and walking except for slightly increased grooming behavior in Aldh2 KI mice (Fig. 2e).

Consequently, we measured adaptive thermogenesis, including cold-induced and diet-induced thermogenesis. For diet-induced thermogenesis, Aldh2 KI mice exhibited significantly lower rectal temperature 30 minutes after HFHSD feeding, indicating impaired diet-induced thermogenesis (Fig. 2f). 
For cold tolerance test, $A / d h 2 \mathrm{KI}$ mice had lower rectal temperature after 12 hours of prolonged cold exposure at $4{ }^{\circ} \mathrm{C}$ (Fig. $2 \mathrm{~g}$ ), indicating cold intolerance. These data suggest that $A / d h 2 \mathrm{KI}$ mice may develop obesity due to reduced energy expenditure resulting from impaired adaptive thermogenesis.

\section{Aldh2 KI mice displayed reduced insulin sensitivity and impaired glucose tolerance}

Since the $A L D H 2$ Glu504Lys mutation is reported to be associated to type 2 diabetes in genome-wide association studies in East-Asians ${ }^{(3)}$, we further examined glucose homeostasis in mice. On HFHSD, insulin tolerance test (ITT) showed significantly higher blood glucose levels with $76.5 \%$ reduction of the inverse area under curve (AUC) of glucose levels in Aldh2 KI mice, indicating increased insulin resistance (Fig. 2h). Intraperitoneal glucose tolerance (i.p.GTT) showed significantly higher blood glucose levels with AUC of glucose levels increased by $109 \%$ in Aldh2 KI mice (Fig. 2i). Oral glucose tolerance test (OGTT) also showed significantly higher blood glucose levels in Aldh2 KI mice; AUC of glucose levels during the test were increased by $111 \%$ in $\mathrm{KI}$ mice, indicating worsened glucose intolerance (Fig. 2j). Aldh $2 \mathrm{KI}$ mice displayed a compensatory increase in insulin secretion after oral glucose load (Fig. 2k). No differences in glucose homeostasis, measured by glucose and insulin tolerance tests, were found between $A / d h 2 \mathrm{KI}$ and WT mice fed a chow diet (Supplementary Fig. 1).

In addition, $A / d h 2 \mathrm{KI}$ mice on the HFHSD showed a trend for increased total cholesterol (204.5 $\pm 7.4 \mathrm{vs.}$ $186.4 \pm 7.5 \mathrm{mg} / \mathrm{dl}, \mathrm{P}=0.09$, Fig. $2 \mathrm{l})$, triglycerides levels $(107 \pm 7.63$ vs. $91.33 \pm 2.75 \mathrm{mg} / \mathrm{dl}, \mathrm{P}=0.10)$ (Fig. $2 \mathrm{~m}$ ) and serum leptin ( $19.6 \pm 2.8$ vs. $12.7 \pm 1.8 \mathrm{mg} / \mathrm{ml}, \mathrm{P}=0.15$, Fig. $2 \mathrm{n})$. However, adiponectin levels were not different between the two groups (Fig. 20).

\section{Aldh2 KI mice had reduced mitochondrial fatty acid oxidation rate and lower respiratory transport chain activity in brown adipose tissue due to 4-HNE adduction}

In view of the reduced adaptive thermogenesis observed in the Aldh2 $\mathrm{KI}$ mice, we compared the expression levels of Ucp1, the major thermogenic protein in BAT and white fat including inguinal and perigonadal fat between the Aldh $2 \mathrm{KI}$ and WT mice by real-time quantitative PCR (RT-qPCR). There was no difference in Ucp 1 expression in the BAT or white fat between the Aldh2 KI and WT mice fed on HFHSD (Fig. 3a). Immunoblots further confirmed that there was no difference in the level of Ucp1 in the BAT, the tissue where Ucp1 was normally expressed (Supplementary Fig. 2).

We next examined the expression of mitochondrial respiratory complex I to V component in BAT. These protein components are essential for the maintenance of the proton gradient in mitochondrial electron transport chain (ETC) and are required for UCP1-mediated thermogenesis. There was no difference in mitochondrial respiratory complex I to V protein components expression in the BAT of Aldh2 KI and WT mice (Supplementary Fig. 3). We also observed no difference in microscopic morphology of BAT, the major thermogenic organ (Supplementary Fig. 4).

Since the ETC protein expression studied showed no difference between Aldh2 KI and WT mice, it implied that the decreased energy expenditure and thermogenesis were likely due to impaired protein functions. 
We found increased protein carbonylation (Fig. 3b) and 4-HNE-adducted mitochondrial proteins (Fig. 3c) in the BAT of the KI mice compared with WT mice. Using liquid chromatography tandem mass spectrometry (LC MS/MS) analysis, we identified 20 4-HNE-adducted BAT mitochondrial proteins in Aldh2 $\mathrm{KI}$ mice and 10 4-HNE adducted mitochondrial proteins in WT mice, with 8 proteins which are present in both Aldh2 KI and WT mice (Fig. 3d, 3e).

Three identified adducted sites were present in proteins involved in fatty acid oxidation (FAO) including 3ketoacyl-CoA thiolase and propionyl-CoA carboxylase. Eleven sites were present in proteins involved in the maintenance of mitochondrial electron transport chain (ETC) including NADPH dehydrogenase (complex I), succinate dehydrogenase (complex II), cyochrome b-1 complex (complex III), ATP synthase, and glycerol-3-phoshphate dehydrogenase. Three adducted sites were present in the MICOS complex, which are essential for the maintenance of inner mitochondrial membrane structure. Hence, we further evaluated the capacity of mitochondrial FAO and ETC to determine the effect of 4-HNE modification of these proteins. The ex vivo FAO rate of the whole BAT tissue isolated from Aldh2 KI mice was significantly reduced by $16 \%(P=0.03)$ compared with WT mice (Fig. 3f). Consistent with the whole BAT tissue, the FAO rate of the cultured primary brown adipocytes isolated from the Aldh2 $\mathrm{KI}$ mice was also significantly reduced by $22 \%(P=0.03$, Fig. $3 g)$. Furthermore, addition of 4 -HNE decreased FAO rate of induced primary brown adipocytes isolated from WT mice in a dose-dependent manner; FAO rates were reduced by $36 \%(P<0.01)$ and $60 \%$ at 5 and $10 \mu \mathrm{M} 4-\mathrm{HNE}$, respectively $(P<0.01)$ (Fig. $3 \mathrm{~h})$. In line with the LC-MS/MS finding, we found a significant reduction in the enzymatic activity of mitochondrial respiratory complexes I, II, and III, but not complex IV, in Aldh2KI mice compared with WT mice (Fig. 3i). Oxygen consumption rate was also significantly decreased in induced primary brown adipocytes isolated from Aldh2 $\mathrm{KI}$ mice compared with WT mice (Fig. $3 \mathrm{j}$ ). Since FAO is the major energy source of thermogenesis and the mitochondrial ETC is essential for maintaining proton gradient required for thermogenesis, the increased protein carbonylations may explain the impaired thermogenesis of $A / d h 2 \mathrm{KI}$ mice.

\section{ALDH2 activator AD-9308/AD-5591 activated wild-type and mutant human ALDH2 enzymatic activity}

AD-9308 is a valine ester prodrug of a potent and selective small molecule ALDH2 activator AD-5591. When administered in vivo, AD-9308 is rapidly converted to AD-5591 by esterase hydrolysis (Fig. 4a). In vitro, AD-5591 treatment increases the catalytic activity of recombinant wild-type and mutant human ALDH2 (Fig. 4b). In vivo, AD-9308 administration showed a good pharmacokinetic profile in mice when administered orally or intravenously with good bioavailability in rodents and dogs (Supplementary Table 2).

Alda- 1 allosterically activates ALDH2 mainly by partially blocking the substrate exit tunnel, thereby accelerating the substrate-enzyme collision without impeding the catalytic sites of Cys302 and Glu268 ${ }^{26}$; Alda-1 also inhibit substrate-induced ALDH2 inactivation by protecting Cys301 and Cys303 oxidation ${ }^{25}$, 30. Using molecular docking, we found that the binding pocket for AD-5591 (Fig. 4C) is close to that of 
Alda-1 (Supplementary Fig. 5a). Similar to Alda-1 (Supplementary Fig. 5b), AD-5591 is bound within a hydrophobic collar by Tyr456, Val458, Lys127, Met124, Gln462, Gly460, Phe459, Val120, and Phe292 of human ALDH2 (Fig. 4d). This binding site leaves the catalytic sites Cys302 and Glu268 unimpeded (Fig. 4e, Supplementary Fig. 5c). Therefore, the mechanism by which AD-5591 activates ALDH2 is very similar to Alda-1. Furthermore, Alda-1 forms a single hydrogen bond with the Asp457 residue ofALDH2. Yet, in our modeling analyses, AD-5591 forms one additional hydrogen bond with Ala461(Fig. 4e), which may explain its higher affinity to ALDH2 than for Alda-1.

\section{ALDH2 activator AD-9308/AD-5591 lowered 4-HNE and attenuated diet-induced obesity, fatty liver, insulin resistance, and glucose intolerance in both WT and Aldh2 KI mice}

In view of the marked reduction in Aldh2 expression in diet-induced obese mice (Supplementary Fig. 6), we next explored whether Aldh2 activation can rescue these obesity-associated phenotypes. From the age of 10 weeks when HFHSD was started, Aldh $2 \mathrm{KI}$ and WT mice were treated with vehicle, $20 \mathrm{mg} / \mathrm{kg} /$ day or $60 \mathrm{mg} / \mathrm{kg} /$ day of AD-9308 by oral gavage for 20 weeks (Fig. 5a). As shown in Fig. 5B and 5C, AD-9308 treatment effectively reduced the diet-induced weight gain in both Aldh $2 \mathrm{KI}$ and WT mice dosedependently. Weight of perigonadal fat, inguinal fat, omental fat, and liver decreased with AD-9308 treatment dose-dependently (Fig. 5d- 5 h). AD-9308 treatment reduced the extent of hepatic steatosis (Fig. $5 \mathrm{i}, 5 \mathrm{j}$ ) and the levels of hepatic triglycerides contents in both Aldh $2 \mathrm{KI}$ and WT mice in a dosedependent manner (Fig. 5k).

Fasting glucose levels were lowered by AD-9308 treatment in both Aldh2 WT and KI mice (Fig. 6a, 6b). AD-9308 treatment effectively reduced insulin resistance in both WT (Fig. 6c) and KI (Fig. 6d) mice and significantly improved glucose tolerance of both WT (Fig. 6e) and KI (Fig. 6f) mice in a dose-dependent manner. AD-9308 also decreased serum 4-HNE levels in both mice groups dose-dependently (Fig. $6 \mathrm{~g}$ ). Figure 6h. shows the summary diagram depicting how reducing 4-HNE by ALDH2 activator AD-9308 ameliorates metabolic disturbances.

Pathological examination reveled no abnormalities in liver or kidney in both Aldh2 WT and KI mice treated with AD-9308 for 20 weeks (Supplementary Table 3). Serum alanine aminotransferase (ALT) and creatinine levels were also not different between groups after AD-9308 treatment for 20 weeks

(Supplementary Fig. 7).

\section{Discussion}

The prevalence of obesity and diabetes mellitus has surged in the past decades and is predicted to continue to rise, especially in East and South Asia ${ }^{1}$. This trend is largely caused by high-calorie diet enriched in fat and sugar, sedentary lifestyle, and their interaction with genetic predisposition 1,2 . Genome-wide association studies have confirmed many genetic loci associated type 2 diabetes and obesity. Specifically, several genetic loci are East Asian-specific. Genetic variants in or near the CDKAL1, $K L F 9, G P 2, A L D H 2$, and ITIH4 genes are associated with obesity $4,32,33$ and genetic variants in or near the 
GDAP1, PTF1A, SIX3, ALDH2, and PAX4 genes ${ }^{3,34}$ are specifically associated with type 2 diabetes in East Asians. Among them, the ALDH2 Glu504Lys mutation that affects 560 million East Asians or nearly $8 \%$ of global population is associated with body mass index, type 2 diabetes, and serum lipids in large metaanalyses of genome-wide association studies.

We demonstrated that Aldh2 knock-in mice carrying the East Asian-specific Glu504Lys mutation were more prone to develop diet-induced obesity, fatty liver, insulin resistance and glucose intolerance than WT mice on HFHSD. Importantly, the ALDH2 activator AD-9308 increased both the catalytic activity of WT and mutant enzyme, reduce serum 4-HNE levels, and effectively alleviated diet-induced obesity, fatty liver, insulin resistance, and glucose intolerance in both $A / d h 2 \mathrm{KI}$ and WT mice in a dose-dependent manner.

BAT is a highly specialized organ enriched in Ucp1 for adaptive thermogenesis. Although Aldh2 KI mice exhibited impaired thermogenesis, unexpectedly, they did not show reduced Ucp1 expression. Instead, we found that several key mitochondrial proteins involved in mitochondrial FAO and ETC were modified by 4HNE adduction. Consistently, previous studies have shown that 4-HNE is mainly generated from oxidation of mitochondrial membranes, with $30 \%$ of 4 -HNE-adducted proteins located within mitochondria 35,36 . Our data further support that the 4-HNE adduction to mitochondrial proteins can be an underlying mechanism of ALDH22 inactivation that leads to the reduction of mitochondrial FAO and ETC function.

Mitochondrial FAO and ETC are required for the maintenance of the proton gradient in the intermembranous space, which is essential for Ucp1-mediated adaptive thermogenesis. In our study, we found that the thermogenic capacity of $A / d h 2 \mathrm{KI}$ mice was reduced. Fatty acids serve as the main fuel suppliers for thermogenesis ${ }^{37}$. It has been estimated that intracellular fatty acids in the BAT contribute $74-84 \%$ of the fuel for thermogenesis upon cold challenge ${ }^{37}$. Cpt1 is the rate-limiting enzyme for the translocation of fatty acids into mitochondria for $\beta$-oxidation. $C p t 1 b^{+/-}$mice developed fatal hypothermia following cold challenge ${ }^{38}$. Adipose-specific Cpt2-knockout mice presented a hypothermic phenotype when exposed to cold ${ }^{39}$. Mice deficient in fatty acid $\beta$-oxidation enzymes, including very-long-chain acyl-CoA dehydrogenase (VLCAD), long-chain acyl CoA dehydrogenase (LCAD), and short-chain acyl CoA dehydrogenase (SCAD) also displayed cold intolerance ${ }^{40-42}$. These data indicate that mitochondrial FAO is critical for adaptive thermogenesis. Furthermore, BAT-specific $L k b 1$-knockout mice, which have reduced expression of ETC complex proteins, also developed impaired thermogenesis ${ }^{43}$, indicating that the integrity of mitochondrial ETC machinery is essential for adaptive thermogenesis. These data strongly support our findings that 4-HNE adduction to mitochondrial proteins involved in mitochondrial FAO and ETC could lead to impaired adaptive thermogenesis.

ALDH2 metabolizes bioactive toxic aldehydes by oxidation. In addition to ALDH2, one of the major pathways to detoxify 4-HNE is mediated through glutathione transferases (GSTs) by conjugation to glutathione. Gsta4 is one of the isoforms of GST with the highest conjugation activity for 4-HNE. Consistent with the present finding, disruption of Gsta4 in mice increased 4-HNE levels and caused obesity in mice ${ }^{44}$. Disruption of the Gst-10 gene, which causes a $50 \%$ increase in 4 -HNE adducts in $C$. 
elegans also resulted in fat accumulation and direct treatment with 4-HNE increases lipid storage in $C$. elegans $^{45}$. Conversely, over-expression of Gst-10 led to 4-HNE reduction and a lean phenotype ${ }^{45}$. Glutathione peroxidase 4 (Gpx4), which resides in the inner mitochondrial membrane, is a scavenger that reduces lipid peroxides. Deficiency of Gpx4 in mice increased the number of 4-HNE adducts and exacerbated glucose intolerance, dyslipidemia, and fatty liver ${ }^{46}$. Collectively, these data indicate that increased aldehydic load due to excessive 4-HNE cause metabolic disorders. Indeed, overexpression of $A L D H 2$ has been shown to decrease both the heart and liver weight and mitochondrial injury in mice fed on a high-fat diet ${ }^{47}$. Interestingly, diet-induced obesity was not observed in these $A L D H 2$ over-expressing mice 47 .

Our study also showed that the Aldh2 KI mice had more severe hepatic steatosis than the WT mice when fed on HFHSD, which can be reversed by AD-9308 treatment. In line with the present results, Alda-1, a prototype of the ALDH2 activator has been shown to alleviate nonalcoholic hepatic steatosis in apolipoprotein E-knockout mice 48 and reversed alcohol-induced hepatic steatosis in animals ${ }^{49}$, supporting activation of ALDH2 also prevent both alcohol and non-alcoholic hepatic steatosis.

Our study has several limitations. First, obesity and related metabolic phenotypes in the Aldh2 KI mice were observed only when fed on HFHSD and not when placed on chow diet, the metabolic phenotypes were not different between the Aldh2 KI and WT mice. Therefore, our findings may not be generalized to the entire population. The HFHSD for mice consists of $58 \%$ calories from fat and $12 \%$ calories from sucrose; while the chow diet is composed of $13 \%$ calories from fat and $3 \%$ calorie from sucrose. According to the Nutrition and Health Surveys in Taiwan (NAHSIT) in 2008, the average calorie intake from fat and sucrose is $33 \%$ and $8 \%$ in adults in Taiwan ${ }^{50}$. In the National Health and Nutrition Examination Survey of U.S adults., the average calorie intake from fat and sucrose is $35 \%$ and $14 \%$ in 201251,52 . The sucrose content is comparable between HFHSD for mice and modern human diet but the fat content of HFHSD is higher than human diet. Even if this concern is relevant, the findings of this study are still substantial given the large number of East Asians (560 million people) carrying this inactivating mutation (Glu504Lys) in the $A L D H 2$ gene. Second, our experiments did not include heterozygous knock-in mice. Third, although the metabolic disorders were normalized in mice treated with AD-9308 for 5 months without pathological changes in the liver and kidney, long-term safety should be formally determined with GLP standard. Last, humans and other mammals have 19 different aldehyde dehydrogenases (ALDH) and at least six are found in the mitochondria ${ }^{30}$. Although AD-9308 does not activate ALDH1A1, ALDH3A1, ALDH4A1, ALDH5A1 and ALDH7A1 members of the ALDH family (data not shown), it is possible that AD-9308 may still have non-specific effect on other ALDHs.

Our study has important clinical implications. We showed that reduced activity of the mitochondrial enzyme, ALDH2, exacerbates obesity-associated pathologies. These pathologies correlate with increased aldehydic load and inactivation of critical mitochondrial proteins involved in FAO and ETC. Significantly, we showed that treatment with an activator of ALDH2, such as AD-9308 prevented these pathologies. These data provide strong evidence for a critical role of toxic aldehydes accumulation and defective 
ALDH2 activity in the pathogenesis of obesity, diabetes, and fatty liver disease. Our study provide a new strategy by targeting ALDH2 for the treatment of obesity-associated metabolic disorders which is rising rapidly in human populations, particularly in the East and South Asia.

\section{Methods}

\section{Animal experiments, administration of diets and drug treatment}

All animal experiments were performed according to institutional ethical guidelines and were approved by the Institutional Animal Care and Use Committee (IACUC), which is accredited by the Association for Assessment and Accreditation of Laboratory Animal Care International (AAALAC). The mice were housed at $22-24{ }^{\circ} \mathrm{C}$ with light: dark cycles of $12: 12$ hours. The mice were fed either a HFHSD (cat. no. D12331, Research Diets) which provided $58 \%$ calorie from fat and $12.5 \%$ calorie from sucrose or chow diet (cat no. 5001, Lab Diet). For the animal experiments, AD-9308 was dissolved in water and was delivered daily to mice by oral gavage. Only male mice were used in this study.

\section{Generation of Aldh2*2/*2knock-in mice}

Aldh2 KI mice carrying the human ALDH2 Glu504Lys mutation were generated by introducing the Glu504Lys mutation within the mouse gene ${ }^{29}$. Both Aldh2 WT controls and Aldh2 KI mice were littermates from mated heterozygous mice.

\section{Glucose and insulin tolerance test}

Oral and intraperitoneal GTT were evaluated after 6 hours of fasting. Tail blood glucose was collected at $0,15,30,45,60,90$, and 120 min after oral gavage or intraperitoneal injection of glucose water $(1 \mathrm{~g} / \mathrm{kg})$ and measured by a glucometer (ACCU-CHECK Performa, Roche). For the ITT, mice were intraperitoneally injected $1 \mathrm{U} / \mathrm{kg}$ insulin (Humulin R, Eli Lilly) after 4 hours of fasting. Tail blood glucose was collected at 0 , $15,30,45,60,90,120$, and 180 min after injection.

\section{Energy expenditure, food intake and physical activity}

Metabolic measurements (food and water intake, locomotor activity, $\mathrm{VO}_{2}$ consumption and $\mathrm{VCO}_{2}$ production) were obtained using the Promethion metabolic phenotyping system (Sable Systems). Monitoring was performed for 5 days after mice have been acclimatized to the cages for 2 days.

\section{Hepatic triglycerides content measurement}

Approximately $80 \mathrm{mg}$ of liver tissue was homogenized in $1800 \mu \mathrm{l}$ of chloroform/methanol (2:1). Then, $360 \mu \mathrm{l}$ of $\mathrm{H}_{2} \mathrm{O}$ was added. The homogenates were centrifuged and the lower $200-\mu \mathrm{l}$ layer was added with $100 \mu \mathrm{l}$ of chloroform with $4 \%$ Triton X-100 and then dried in a chemical hood. The dried pellet was redissolved with $200 \mu \mathrm{l}$ of $\mathrm{H}_{2} \mathrm{O}$ for determination of triglyceride concentrations with Wako TG LabAssay kit (cat. no. 290-63701, Wako) 


\section{Cold tolerance test and diet-induced thermogenesis test}

For the cold tolerance test, 24-week-old mice with matched average body weight from the two groups were placed individually on HFHSD in a $4^{\circ} \mathrm{C}$ chamber. The rectal temperature of the mice was measured after $0,1,2,3,4,5,6,12$, and 18 hours. For the diet-induced thermogenesis test, 24-week-old mice were fasted overnight for 18 hours. Then, their rectal temperature was measured at $0,15,30,60,120,180$, and 240 min after HFHSD refeeding.

\section{Real-time quantitative PCR (RT-qPCR)}

RT-qPCR was performed using SYBR green reagent (cat. no. 11203ES08, YEASEN). The primer sequences for mouse Ucp 1 and Ppia is list in Table S1. RT-qPCR reactions were performed using an ABI 7900HT FAST (Applied Biosystems). All qPCR reactions were run in duplicate for each sample.

\section{Primary brown adipocyte culture}

BAT from Aldh2 WT and KI mice aged 4 weeks was minced and digested by type I collagenase (cat. no. 17018029, Thermo Fisher Scientific) in HEPES buffer. The stromal vascular fraction (SVF) was obtained by centrifugation and cultured in Dulbecco's modified Eagle medium:nutrient mixture F-12 (DMEM/F-12) (cat. no. 12500062, HyClone) supplemented with 10\% FBS (cat. no. 04-001-1A, Biological Industries) and $1 \subseteq$ antibiotic/antimycotic solution (cat. no. SV30079.01, HyClone). For cell differentiation, preadipocytes were cultured in differentiation medium containing 10\% FBS, $0.5 \mathrm{mM}$ isobutyl-methylxanthine, $1 \mu \mathrm{g} / \mathrm{ml}$ insulin, $5 \mu \mathrm{M}$ dexamethasone, $1 \mathrm{nM}$ T3, and $125 \mu \mathrm{M}$ indomethacin for 2 days. Next, cells were maintained in the medium containing DMEM/F12 with $10 \% \mathrm{FBS}, 1 \mu \mathrm{g} / \mathrm{ml}$ insulin, and $1 \mathrm{nM}$ T3. The medium was changed every 2 days.

\section{Isolation of mitochondria from brown adipose tissue}

BAT was removed from Aldh2 IWT and KI mice fed a HFHSD for 5 weeks. Isolation of mitochondria was performed according to published protocols ${ }^{53}$. Briefly, BAT was homogenized in $10 \% \mathrm{w} / \mathrm{v}$ of ice-cold mitochondrial isolation buffer by a Dounce homogenizer. The pellets were separated by centrifugation, and then resuspended in SET buffer ( $0.25 \mathrm{M}$ sucrose, $1 \mathrm{mM}$ EDTA, and $10 \mathrm{mM}$ Tris- $\mathrm{HCl}, \mathrm{pH}$ 7.4) for the fatty acid oxidation assay and LC-MS/MS analysis or in BES-sucrose buffer $(0.25 \mathrm{M}$ sucrose, $0.1 \mathrm{M} \mathrm{KCl}$, and $20 \mathrm{mM} \mathrm{BSE}, \mathrm{pH}$ 7.2) for the mitochondrial complex activity assay.

\section{Fatty acid oxidation (FAO) assay}

FAO measurements were performed using labeled ${ }^{3} \mathrm{H}$-palmitic acid and ${ }^{3} \mathrm{H}_{2} \mathrm{O}$ production was assessed as previously described ${ }^{54}$. For FAO rate of differentiated primary cells, the capture of ${ }^{3} \mathrm{H}_{2} \mathrm{O}$ was measured after a 2-hour incubation with $5 \mathrm{mM}$ palmitate/BSA buffer including $0.5 \mu \mathrm{Ci}\left[{ }^{3} \mathrm{H}\right]$-palmitate (cat. no. PKNET043001MC, PerkinElmer) in the presence of $1 \mathrm{mM}$ carnitine. For FAO rate of whole BAT, the isolated mitochondria were placed in a 24-well plate and added incubated with reaction buffer $(100 \mathrm{mM}$ sucrose, $10 \mathrm{mM}$ Tris- $\mathrm{HCl}, 5 \mathrm{mM} \mathrm{KH}_{2} \mathrm{PO}_{4}, 0.2 \mathrm{mM}$ EDTA, $80 \mathrm{mM} \mathrm{KCl}, 1 \mathrm{mM} \mathrm{MgCl} 2,2 \mathrm{mM}$ L-carnitine, $0.1 \mathrm{mM}$ malate, $0.05 \mathrm{mM}$ coenzyme A, $2 \mathrm{mM}$ ATP, $1 \mathrm{mM}$ dithiothreitol, and 7\% BSA/5 mM palmitate/0.01 $\mu \mathrm{Ci} / \mu \mathrm{l}$ 
$\left[{ }^{3} \mathrm{H}\right.$-palmitate) at $37^{\circ} \mathrm{C}$ for $60 \mathrm{~min} .{ }^{3} \mathrm{H}_{2} \mathrm{O}$ was isolated by oil-water separation with chloroform, methanol and $\mathrm{KCl} / \mathrm{HCl}$. The average counts per minute (CPM) were measured using a liquid scintillation counter.

\section{Biotin hydrazide staining for carbonylated protein}

For detection of carbonylated protein, samples were chemically reduced by $\mathrm{NaBH}_{4}$ and then incubated with $0.5 \mathrm{mM}$ EZ-link biotin hydrazide (ca. no.21339, Pierce) for 1 hour. After coupling, the samples were separated by $10 \%$ SDS-PAGE gel and transferred to PVDF membrane. The membrane was blocked with $10 \%$ skim milk in PBS containing $0.05 \%$ Tween-20 (PBST) at $4^{\circ} \mathrm{C}$ overnight and then incubated with Streptavidin-HRP (cat no. 890803, BD Biosciences) for 1 hour at room temperature. Chemiluminescence signals were developed with HRP substrate (cat. no.WBLUR0500, Millipore).

Plasmid construction, expression and purification of human ALDH2 in Escherichia coli

E. coli BL21 (DE3) was transformed with pTrcHi-WT ALDH2 and pTrcHi-KI ALDH2 ${ }^{29}$. The transformants were cultured and then induced to express recombinant proteins using $0.4 \mathrm{mM}$ IPTG for 16-18 hours at $25^{\circ} \mathrm{C}$. The cells were harvested by centrifugation and broken in lysis buffer by sonication on ice. The recombinant ALDH2 protein was purified with NI-NTA resin (cat. no. 88222, ThermoFisher) following the user manual.

\section{ALDH2 enzymatic activity assays}

ALDH2 activity was measured by monitoring the reduction rate of $\mathrm{NAD}^{+} / \mathrm{min}$ at $340 \mathrm{~nm}$ and $25^{\circ} \mathrm{C}$. Enzyme activity was assayed in $100 \mu \mathrm{l}$ of reaction mixtures containing $50 \mathrm{mM} \mathrm{Na}{ }_{4} \mathrm{P}_{2} \mathrm{O}_{7}(\mathrm{pH}$ 9.5), $0.01 \%$ $\mathrm{BSA}, 10 \mathrm{mM} \mathrm{NAD}^{+}, 50 \mu \mathrm{M}$ acetaldehyde and recombinant ALDH2 with or without $100 \mu \mathrm{M}$ AD-5591 or Alda-1. One enzymatic activity unit was defined as the reduction of $1 \mu \mathrm{mol} \mathrm{NAD}{ }^{+}$per min by $1 \mathrm{mg}$ of human ALDH2 protein.

\section{Molecular docking}

To visualize the interaction between the ALDH2 with activators Alda- 1 and AD-5591, the X-ray structure of human ALDH2 (PDB ID: 1NZX) and NAD ${ }^{+}$were used. Ligand energy was minimized using the PyRx program before docking ${ }^{55}$. Three-dimensional models are generated by using the PyMOL program ${ }^{56}$ and the $2 \mathrm{D}$ protein-ligand interaction diagrams were generated by the LigPlot + program ${ }^{57}$.

\section{LC - MS/MS analysis of 4-HNE-modified proteins}

The protein samples were prepared with in-solution trypsin digestion. The peptides were desalted with C18 Zip Tip (Millipore, USA) and dry by vacuum centrifugation. The desalted and dried peptides were resuspended in $0.1 \%$ formic acid.

LC-MS/MS analysis was performed on a NanoACQUITY UPLC system (Waters, USA) coupled to a highresolution mass spectrometer (QE HF-X, Thermo Fisher Scientific). The peptides were injected into a trap column (Symmetry C18, $2 \mathrm{~cm} \times 75 \mu \mathrm{m}$ i.d.) and then separated in a $25 \mathrm{~cm} \times 75 \mu \mathrm{m}$ i.d. BEH130 C18 
column (Waters, USA) on a gradient from $0-85 \%$ buffer $\mathrm{B}$ (buffer $\mathrm{A}, 0.1 \% \mathrm{FA} \mathrm{H}_{2} \mathrm{O}$; buffer $\mathrm{B}, 0.1 \%$ formic acid in acetonitrile). The mass spectrometer was operated in data-dependent mode with the following acquisition cycle: an MS scan (m/z 350-1600) recorded at resolution $R=60,000$ and MS/MS scans recorded at resolution $\mathrm{R}=15,000$, which were acquired by HCD fragmentation with collision energy of 28 .

MS/MS spectra were searched with the Mascot engine (v2.6, Matrix Science) against the UniProtKB mouse protein database using the following parameters: a precursor peptide mass tolerance of $20 \mathrm{ppm}$ and an MS/MS fragment tolerance of $0.02 \mathrm{Da}$ with a maximum of two missed cleavage sites. The following modifications were made to the peptides: static carbamidomethylation on cysteine, variable oxidation on methionine, variable deamidation of asparagine or glutamine, and various 4-HNE modifications on cysteine, histidine and lysine. The cut-off threshold for acquiring significant peptide-tospectrum matches was $\mathrm{P}<0.05$.

\section{Mitochondrial respiratory chain complex activity assay}

The mitochondrial complex spectrophotometric assays were carried out using published protocols ${ }^{58,59}$. Complex I and complex II activities were measured spectrophotometrically by examining the decrease in absorbance due to the reduction of 2,6-dichlorophenolindophenol (DCPIP) at $600 \mathrm{~nm}$. Activity was expressed in nanomoles of DCPIP reduction/min/mg protein $\left(E=19.1 \mathrm{mmol}^{-1} \cdot \mathrm{cm}^{-1}\right)$. Complex IIIspecific activity and complex IV-specific activity were measured by monitoring the reduction of oxidized cytochrome $C$ (III) and oxidation of reduced cytochrome $C$ (II) at $550 \mathrm{~nm}$, respectively. The activity is expressed as a nanomole of reduced cytochrome of oxidized cytochrome $\mathrm{C} / \mathrm{min} / \mathrm{mg}$ protein $(\mathrm{E}=$ $\left.18.5 \mathrm{mmol}^{-1} \cdot \mathrm{cm}^{-1}\right)$.

\section{Immunoblots to detect 4-HNE-adducted proteins}

Samples prepared with Laemmli sample buffer and were separated by $10 \%$ SDS-PAGE gel and transferred to PVDF membrane. The membrane was blocked with $10 \%$ skim milk in PBST and incubated at $4^{\circ} \mathrm{C}$ overnight with anti-4-HNE antibody (1:1000; cat. no. PAB1295, Abnova) and then with secondary antibodies with HRP (1:10000; cat. no. GTX26721, GeneTex).

\section{Oxygen consumption rate (OCR)}

Stromal vascular fraction was isolated from BAT of 4-week-old Aldh2 WT and KI mice and seeded to Seahorse XF24 v7 cell culture plates (Agilent) at approximate density of 20,000 cells per well and was then differentiated to primary brown adipocyte as described above. On day7 of differentiation, cells were washed once with Seahorse medium and maintained in Seahorse medium in $\mathrm{CO}_{2}$-less incubators. Basal OCR were determined by three measurements, followed by three measurements after each injection drug: oligomycin $(2 \mu \mathrm{M})$, FCCP $(2 \mu \mathrm{M})$, Rotenone/Antimycin A $(0.5 \mu \mathrm{M})$ using Seahorse XFe24 Analyzer (Agilent). OCR measurements of each stage were normalized to the protein concentration.

\section{Serum biochemistry}


Blood was collected from mice fasted for 4 hours. Fasting plasma adiponectin (cat. no. ab108785, Abcam), leptin (cat. no. DY498, R\&D), and insulin (cat. no. 10-1247-01, Mercodia), 4-HNE (cat. no. EELM2677, Elabscience) concentrations were measured using ELISA kits. Plasma triglycerides, and total cholesterol levels were measured using the FUJI DRI-CHEM clinical chemistry analyzer.

\section{Statistical analyses}

Two-tailed independent $t$-tests were used for comparing two independent groups. The Wilcoxon rank-sum test was used to compare data from RT-qPCR. Statistical analyses were conducted using GraphPad Prism 5.0. All P-values $<0.05$ were considered statistically significant.

\section{Data availability}

All data associated with this study are available in the main text or the supplementary materials

\section{Declarations}

\section{Competing interests}

D.M-R, C.H.C and W.Y. are co-inventors on several issues patents on "Modulators of aldehyde dehydrogenase activity and methods of use thereof", patent Numbers: US 10227304, US 9670162, US 9370506, US 9345693, US 8906942, US 8772295, US 8389522, and US 8354435. W.Y. is an employee and shareholder of Foresee Pharmaceuticals, Co., Ltd. W.Y. is a co-inventor of issued patent US 9879036 "Modulators of aldehyde dehydrogenase activity and methods of use thereof". Other authors declared no competing interests.

\section{Author contributions}

H.L.L. performed the LC-MS/MS analyses, immunoblots, RT-qPCR, histology examinations, and enzyme activity assays. J.Y.H. and W.L.S. performed the animal experiments. Y.C.C and J.Y.N. performed ELISA. Z.Z.D. performed histology examinations. M.L.H. and Y.T.T. conducted the pathological examinations. M.L.H. performed molecular docking. C.C.L. performed the $\beta$-oxidation assay and LC-MS/MS experiments. T.Y.L. performed the OCR measurement. W.Y designed and synthesized AD9308 and AD5591 and conducted pharmacokinetic studies. F.A.L. performed the LC-MS/MS experiments. H.L.C. performed indirect calorimetry. L.Y.C. drew summary graphs. Y.C.C. H.L.L, W.Y, C.H.C. D.M.R. and L.M.C. wrote the manuscript. L.M.C. D.M.R. and Y.C.C. conceived the study and participated in the design and interpretation of all experiments.

\section{Acknowledgments}


We thank the Proteomics Core Facility at Institute of Biomedical Sciences of Academia Sinica and the Metabolomics Core Facility in the Scientific Instrument Center of Academia Sinica for proteomic studies. We thank the support from Taiwan Animal Consortium, Taiwan Mouse Clinic, and the Phenotyping Service and Pathology Core Facility of National Laboratory Animal Center of Taiwan for technical support. We thank Foresee Pharmaceuticals Co., Ltd., Taiwan for the synthesis and provision of AD-9308 and AD-5591.This study was supported by the Ministry of Science and technology of Taiwan (101-2314B-002-158-MY3, 105-2314-B-002-105-MY3, 106-2321-B-002-040, and 107-2321-B-002 -067), National Taiwan University and National Taiwan University Hospital (UN105-0072 and UN109-008) to L.M.C. and Y.C.C., The New Century Health Care Promotion Foundation Grants to YCC, SPARK Translational Research Program 2013 grant to C.C.H., and NIAAA11147 grant from the NIH to D.M-R.

\section{References}

1. The International Diabetes Federation. Diabetes Atlas 9th edition 2019 report https://diabetesatlas.org/en/sections/worldwide-toll-of-diabetes.html

2. Dietrich, S., et al. Gene-lifestyle interaction on risk of type 2 diabetes: A systematic review. Obes. Rev. 20, 1557-1571 (2019).

3. Li, H., et al. Refined geographic distribution of the oriental ALDH2*504Lys (nee 487Lys) variant. Ann. Hum. Genet. 73, 335-345 (2009)

4. FarrÈs, J. X., et al. Effects of changing glutamate 487 to lysine in rat and human liver mitochondrial aldehyde dehydrogenase. A model to study human (Oriental type) class 2 aldehyde dehydrogenase. J. Biol.Chem. 269, 13854-13860 (1994).

5. Chen, Y.C., Peng, G. S., Wang, M.F., Tsao, T.P. \& Yin, S.J. Polymorphism of ethanol-metabolism genes and alcoholism: correlation of allelic variations with the pharmacokinetic and pharmacodynamic consequences. Chemm. Biol. Interact. 178, 2-7 (2009).

6. Kim, S.W., et al. The role of acetaldehyde in human psychomotor function: a double-blind placebocontrolled crossover study. Biol. Psychiatry. 67, 840-845 (2010).

7. Kimura, M., A. \& Higuchi, Y.S. Aldehyde dehydrogenase-2 as a therapeutic target. Expert. Opin. Ther. Targets. 23, 955-966 (2019).

8. Cadoni, G.., et al. A review of genetic epidemiology of head and neck cancer related to polymorphisms in metabolic genes, cell cycle control and alcohol metabolism. Acta. Otorhinolaryngol. Ital. 32, 1-11 (2012).

9. Spracklen, C. N., et al. Identification of type 2 diabetes loci in 433,540 East Asian individuals. Nature $582,240-245$ (2020).

10. Wen, W., et al. Meta-analysis of genome-wide association studies in East Asian-ancestry populations identifies four new loci for body mass index. Hum. Mol. Genet. 23, 5492-5504 (2014).

11. Lu, X., et al. Consortium, Exome chip meta-analysis identifies novel loci and East Asian-specific coding variants that contribute to lipid levels and coronary artery disease. Nat. Genet. 49, 1722-1730 (2017). 
12. Wang, T., et al. Effects of Obesity Related Genetic Variations on Visceral and Subcutaneous Fat Distribution in a Chinese Population. Sci. Rep. 6, 20691 (2016).

13. Oniki, K., et al. The longitudinal effect of the aldehyde dehydrogenase $2 \star 2$ allele on the risk for nonalcoholic fatty liver disease. Nutr. Diabetes. 6, e210-e210 (2016).

14. Klyosov, A.A., Rashkovetsky, L.G., Tahir, M.K. \& Keung, W.M. Possible Role of Liver Cytosolic and Mitochondrial Aldehyde Dehydrogenases in Acetaldehyde Metabolism. Biochemistry. 35, 4445-4456 (1996).

15. Michael Lieberman, Allan D. Marks. Marks' Basic Medical Biochemistry: A Clinical Approach. 3rd edition. Chapter 25. (Lippincott Williams \& Wilkins, 2009)

16. Zhang, J., Lioy, P.J. \& He, Q. Characteristics of aldehydes: concentrations, sources, and exposures for indoor and outdoor residential microenvironments. Environ. Sci. Technol. 28, 146-152 (1994).

17. O'Brien, P.J., Siraki, A.G., \& Shangari, N. Aldehyde sources, metabolism, molecular toxicity mechanisms, and possible effects on human health. Crit. Rev. Toxicol. 35, 609-662 (2005).

18. Bach, C.,et al. Effect of temperature on the release of intentionally and non-intentionally added substances from polyethylene terephthalate (PET) bottles into water: Chemical analysis and potential toxicity. Food. Chem. 139, 672-680 (2013).

19. International Agency for Research on Cancer. IARC Monographs on the Identification of Carcinogenic Hazards to Humans. Agents Classified by the IARC Monographs, Volumes 1-125.

https://monographs.iarc.fr/agents-classified-by-the-iarc/

20. Salaspuro, M. Acetaldehyde as a common denominator and cumulative carcinogen in digestive tract cancers. Scand. J. Gastroenterol. 44, 912-925 (2009).

21. Brooks, P.J., Enoch, M.A., Goldman, D, Li, T.L \& Yokoyama, A. The alcohol flushing response: an unrecognized risk factor for esophageal cancer from alcohol consumption. PLoS. Med. 6, e50 (2009). 22. Langevin,.., Crossan, G. P., Rosado, I.V., Arends, M. J. \& Patel, K. J. Fancd2 counteracts the toxic effects of naturally produced aldehydes in mice. Nature 475, 53-58 (2011).

23. Riahi Y., Cohen, G., Shamni, O. \& Sasson, S. Signaling and cytotoxic functions of 4-hydroxyalkenals. Am. J. Physiol. Endocrinol. Metab. 299, E879-E886 (2010).

24. Xiao, M., Zhong, H., Xia, L., Tao, Y. \& Yin, H. Pathophysiology of mitochondrial lipid oxidation: Role of 4-hydroxynonenal (4-HNE) and other bioactive lipids in mitochondria. Free. Radic. Biol. Med. 111, 316-327 (2017).

25. Chen, C.H., et al. Activation of aldehyde dehydrogenase-2 reduces ischemic damage to the heart. Science 321, 1493-1495 (2008).

26. Perez-Miller, S., et al. Alda-1 is an agonist and chemical chaperone for the common human aldehyde dehydrogenase 2 variant. Nat. Struct. Mol. Biol. 17, 159-164 (2010).

27. Tsai, S.H., et al. Aldehyde dehydrogenase 2 protects against abdominal aortic aneurysm formation by reducing reactive oxygen species, vascular inflammation, and apoptosis of vascular smooth muscle cells. FASEB. J. 34: 9498-9511.(2020)

28. Hsu, L.A., et al. Aldehyde dehydrogenase 2 ameliorates chronic alcohol consumption-induced atrial fibrillation through detoxification of 4-HNE. Int. J. Mol. Sci. 21:6678. (2020)

29. Joshi, A.U., et al. Aldehyde dehydrogenase 2 activity and aldehydic load contribute to 
neuroinflammation and Alzheimer's disease related pathology. Acta. Neuropathol. Commun. 7:190 (2019) 30. Zambelli, V. O., Gross, E. R., Chen, C.H., Gutierrez, V. P., Cury Y. \& Daria, M-R. Aldehyde dehydrogenase-2 regulates nociception in rodent models of acute inflammatory pain. Sci. Transl. Med. 6:251 ra118 (2014). 30. Chen, C.H., Ferreira, J. C. Gross, E. R. \& Daria, M-R. Targeting aldehyde dehydrogenase 2: new therapeutic opportunities. Physiol. Rev. 94, 1-34 (2014).

31. Yang, W., Yu, Y.T. \& Jiang, C. Mitochondrial aldehyde dehydrogenase-2 binding compounds and methods of use thereof. U.S. Patent 9,879,036 B2, issued Jan. 30, 2018.

32. Wen, W., et al. Meta-analysis identifies common variants associated with body mass index in east Asians. Nat. Genet. 44, 307-311 (2012).

33. Okada, Y., et al. Common variants at CDKAL1 and KLF9 are associated with body mass index in east Asian populations. Nat. Genet. 44, 302-306 (2012).

34. Cho Y.S., et al. Meta-analysis of genome-wide association studies identifies eight new loci for type 2 diabetes in east Asians. Nat. Genet. 44, 67-72 (2011).

35. Zhao, Y., et al. Redox proteomic identification of HNE-bound mitochondrial proteins in cardiac tissues reveals a systemic effect on energy metabolism after doxorubicin treatment. Free. Radic. Biol. Med. 72, 55-65 (2014).

36. Poli, G., Schaur R. J., Siems W. G. \& Leonarduzzi, G. 4-hydroxynonenal: a membrane lipid oxidation product of medicinal interest. Med. Res. Rev. 28, 569-631 (2008).

37. Labbé, S. M., et al. In vivo measurement of energy substrate contribution to cold-induced brown adipose tissue thermogenesis. FASEB. J. 29, 2046-2058 (2015).

38. Ji. S., et al. Homozygous carnitine palmitoyltransferase $1 \mathrm{~b}$ (muscle isoform) deficiency is lethal in the mouse. Mol. Genet. Metab. 93, 314-322 (2008).

39. Lee, J. Ellis, J. M. \& Wolfgang, M.J. Adipose Fatty Acid Oxidation Is Required for Thermogenesis and Potentiates Oxidative Stress-Induced Inflammation. Cell. Rep. 10, 266-279 (2015).

40. Schuler, A.M., et al. Synergistic heterozygosity in mice with inherited enzyme deficiencies of mitochondrial fatty acid beta-oxidation. Mol. Genet. Metab. 85, 7-11 (2005).

41. Thorpe,C.\& Kim, J.J. P. Structure and mechanism of action of the Acyl-CoA dehydrogenases1. FASEB. J. 9, 718-725 (1995).

42. Gregersen, N., et al. Mutation analysis in mitochondrial fatty acid oxidation defects: Exemplified by acyl-CoA dehydrogenase deficiencies, with special focus on genotype-phenotype relationship. Hum. Mutat. 18, 169-189 (2001).

43. Masand, R., et al. Proteome Imbalance of Mitochondrial Electron Transport Chain in Brown Adipocytes Leads to Metabolic Benefits. Cell. Metab. 27, 616-629.e614 (2018).

44. Singh, S.P., et al. Role of the Electrophilic Lipid Peroxidation Product 4-Hydroxynonenal in the Development and Maintenance of Obesity in Mice. Biochemistry. 47, 3900-3911 (2008).

45. Singh S. P., Niemczyk, M., Zimniak, L.\& Zimniak, P. Fat accumulation in Caenorhabditis elegans triggered by the electrophilic lipid peroxidation product 4-hydroxynonenal (4-HNE). Aging (Albany NY) 1 , 68-80 (2008).

46. Katunga, L.A., et al. Obesity in a model of gpx4 haploinsufficiency uncovers a causal role for lipidderived aldehydes in human metabolic disease and cardiomyopathy. Mol. Metab. 4, 493-506 (2015). 
47. Wang, S., et al. ALDH2 protects against high fat diet-induced obesity cardiomyopathy and defective autophagy: role of CaM kinase II, histone H3K9 methyltransferase SUV39H, Sirt1, and PGC-1a deacetylation. Int. J. Obes .(Lond) 42, 1073-1087 (2018).

48. Stachowicz, A., et al. Mitochondrial Aldehyde Dehydrogenase Activation by Alda-1 Inhibits Atherosclerosis and Attenuates Hepatic Steatosis in Apolipoprotein E Knockout Mice. J. Am. Heart. Assoc. 3, e001329 (2014).

49. Zhong, W., et al. Pharmacological activation of aldehyde dehydrogenase 2 by Alda-1 reverses alcoholinduced hepatic steatosis and cell death in mice. J. Hepatol. 62, 1375-1381 (2015).

50. Pan, H., et al. Diet and health trends in Taiwan: comparison of two nutrition and health surveys from 1993-1996 and 2005-2008. Asia. Pac. J. Clin. Nutr. 20:238-50 (2011)

51. Powell, E.S., Smith-Taillie, L.P. \& Popkin, M. Added sugars intake across the distribution of US children and adult consumers: 1977-2012. J. Acad. Nutr. Diet. 116, 1543-1550.e1541 (2016).

52. Rehm, C.D., Peñalvo, J.L., Afshin, A. \& Mozaffarian, D. Dietary intake among US adults, 1999-2012. JAMA. 315:2542-53 (2016).

53. Sun, K.C., et al. Brown adipose tissue derived VEGF-A modulates cold tolerance and energy expenditure. Mol. Metab. 3, 474-483 (2014).

54. Saddik, M. \& Lopaschuk, G. D. Myocardial triglyceride turnover and contribution to energy substrate utilization in isolated working rat hearts. J. Biol. Chem. 266, 8162-8170 (1991).

55. Dallakyan, S. \& Olson, A. J. Small-molecule library screening by docking with PyRx. Methods. Mol. Biol. 1263, 243-250 (2015).

56. Rigsby, R. E. \& Parker, A. B. Using the PyMOL application to reinforce visual understanding of protein structure. Biochem. Mol. Biol.Edu. 44, 433-437 (2016).

57. Laskowski, A. \& Swindells, M. B. LigPlot+: Multiple Ligand-Protein Interaction Diagrams for Drug Discovery. J Chem. Inf. Model. 51, 2778-2786 (2011).

58. Janssen, F. J., et al. Spectrophotometric Assay for Complex I of the Respiratory Chain in Tissue Samples and Cultured Fibroblasts. Clin. Chem. 53, 729-734 (2007).

59. Spinazzi, M., Casarin, A., Pertegato,V., Salviati, L. \& Angelini,C. Assessment of mitochondrial respiratory chain enzymatic activities on tissues and cultured cells. Nat. Protocols 7, 1235-1246 (2012).

\section{Figures}


a

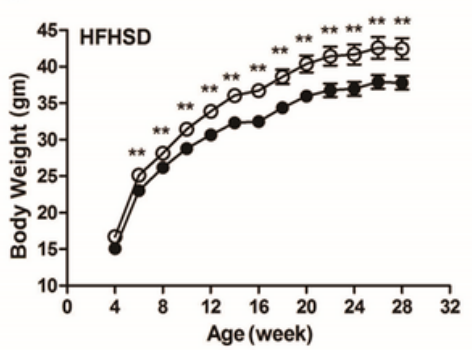

b

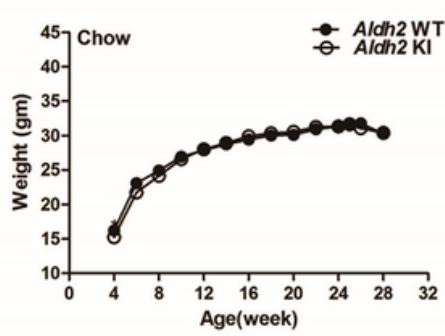

C

d

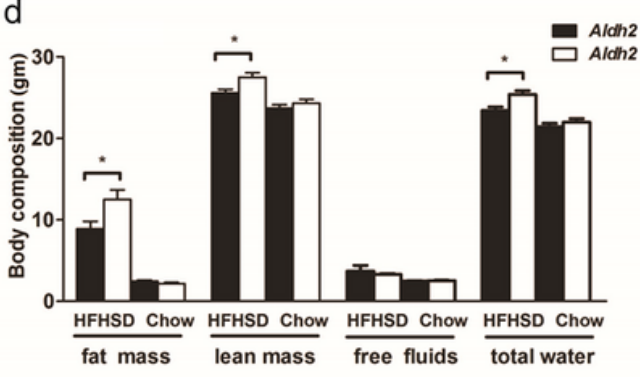

e

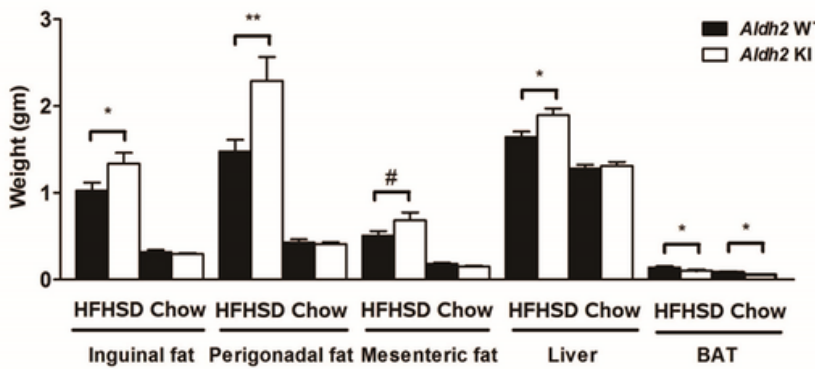

h

Aldh2 WT

Aldh2 KI
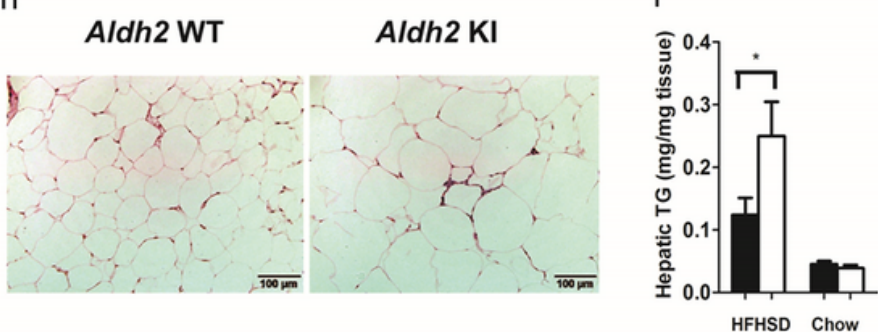

j

WT KI $\quad$ KI
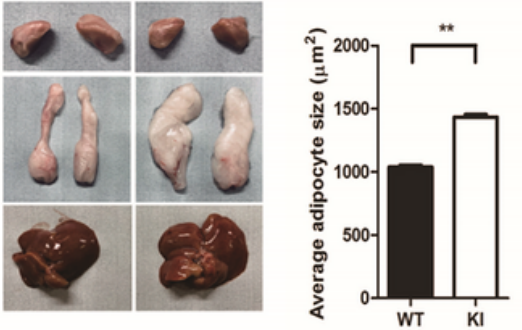

Aldh2 WT

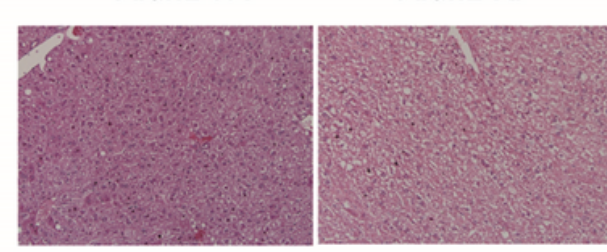

g

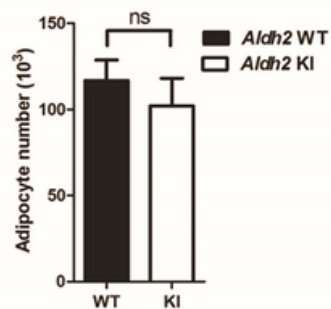

k

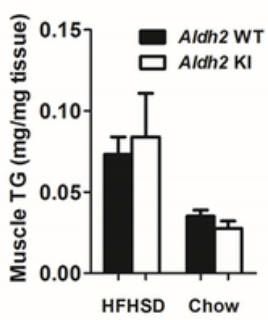

\section{Figure 1}

Body weight of Aldh2 WT and KI mice on (a) HFHSD ( $n=24: 21)$ and (b) chow ( $n=15: 19)$. (c) Weights of inguinal, perigonadal, and mesenteric fat, the liver, and brown adipose tissue (BAT) of the Aldh2 WT and KI mice on HFHSD ( $n=67: 48)$ and chow ( $n=10: 12)$. (d) Body composition of Aldh2 WT and KI mice on a HFHSD $(n=12: 20)$ and chow $(n=15: 19)$. (e) Gross appearance if mice, BAT, perigonadal fat and liver. ( $f$ ) Average adipocyte size and (g) adipocyte number in the perigonadal fat pad of the Aldh2 WT and KI mice on HFHSD ( $n=24: 16)$. (h) H\&E stain of the perigonadal fat in Aldh2 WT and KI mice on HFHSD. (i) Hepatic triglyceride content of the Aldh2 WT and KI mice on HFHSD ( $n=23: 20)$ and chow diet $(n=10: 8)$. (j) Representative H\&E staining of livers from the Aldh2 WT and KI mice. (k) Muscle triglyceride content in the Aldh2 WT and KI mice on HFHSD $(n=23: 20)$ and chow diet $(n=10: 8)$. ${ }^{*}<0.05, * * P<0.01$. 
a

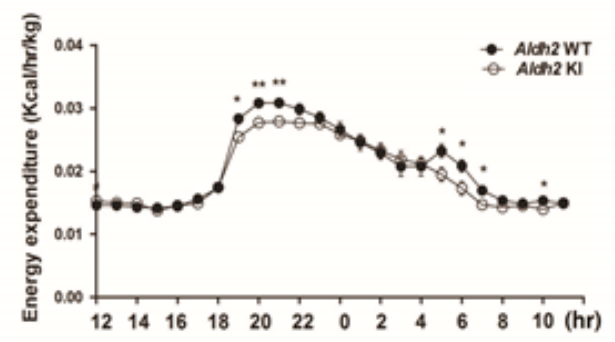

e

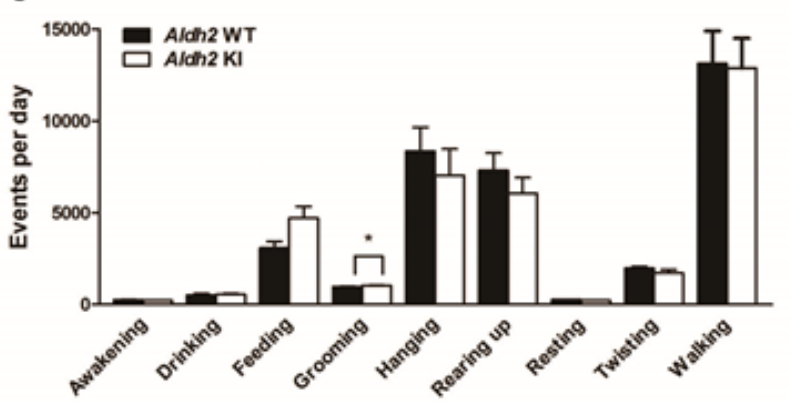

g

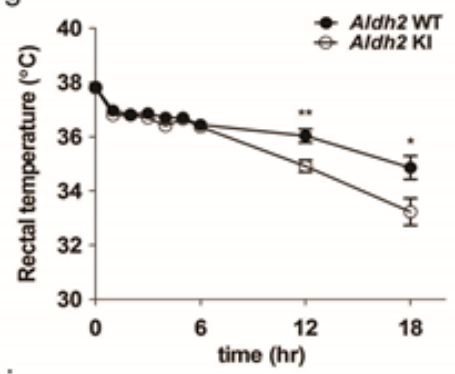

j
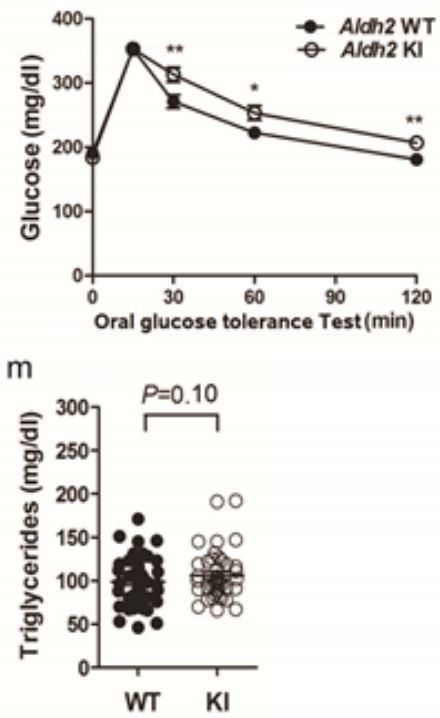

$\mathrm{h}$

k

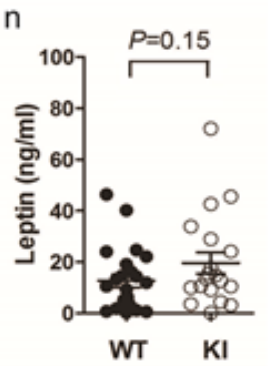

b c
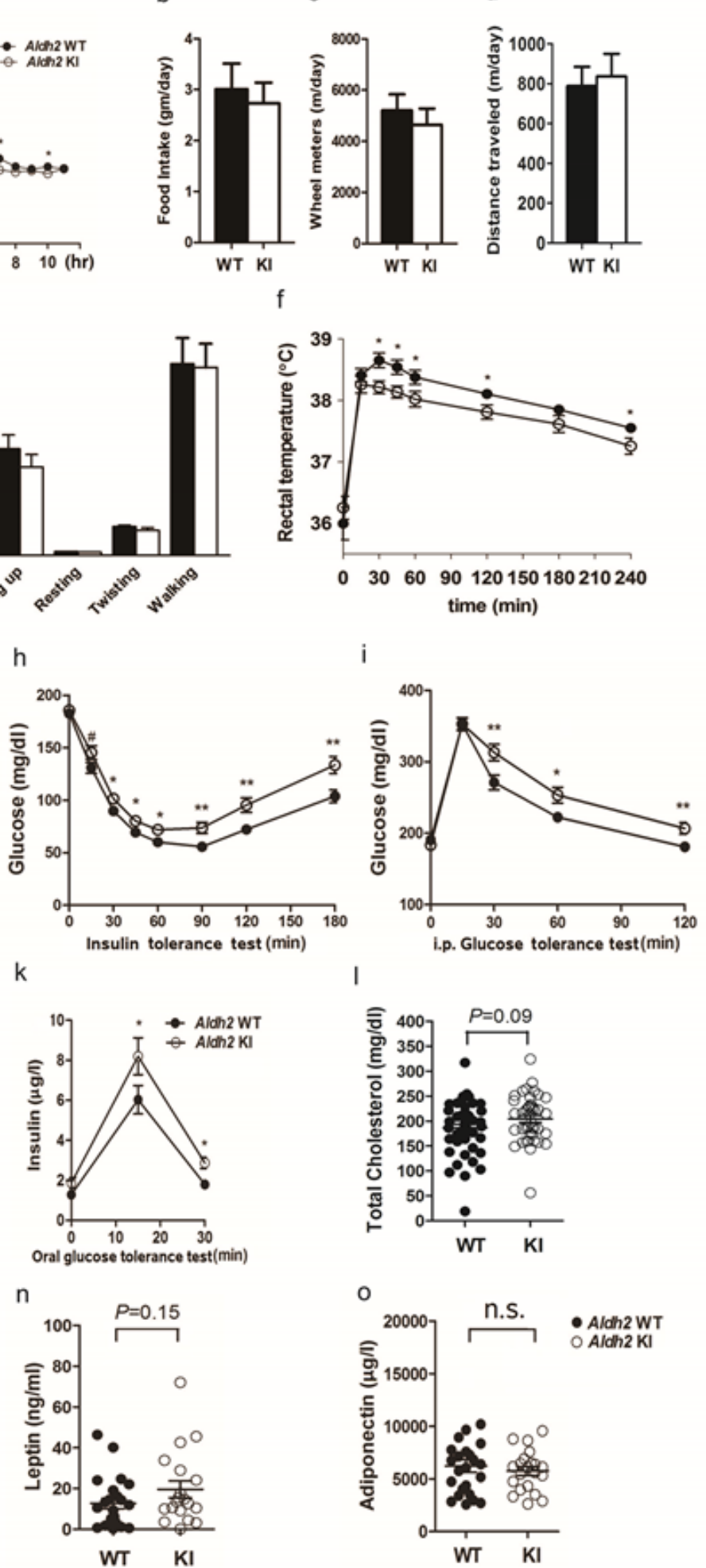

Figure 2

(a) Energy expenditure ( $n=12: 12)$, (b) food intake $(n=12: 12)$, (c) wheel rotations $(n=12: 12)$, (d) distance traveled ( $n=6: 6)$, and (e) behaviors monitored by HomeCage systems $(n=6: 6)$ of the Aldh2 WT and KI mice on HFHSD. (f) Diet-induced thermogenesis and (g) cold-induced thermogenesis of the Aldh2 WT and $\mathrm{KI}$ mice on a HFHSD ( $n=28: 18)$. (h) Glycemic levels during the insulin sensitivity test $(n=50: 44)$ and (i) intraperitoneal glucose tolerance test $(n=50: 44)$, and (j) glycemic levels during the oral glucose tolerance 
test of Aldh2 WT and KI mice on HFHSD (n=50:44). (k) Insulin levels following oral glucose load $(n=45: 36)$, and fasting serum $(I)$ total cholesterols, $(m)$ triglycerides, $(n)$ leptin, and $(0)$ adiponectin $(n=24: 21)$ of Aldh2 WT and KI mice on HFHSD. ${ }^{*} P<0.05, * * P<0.01$.

a

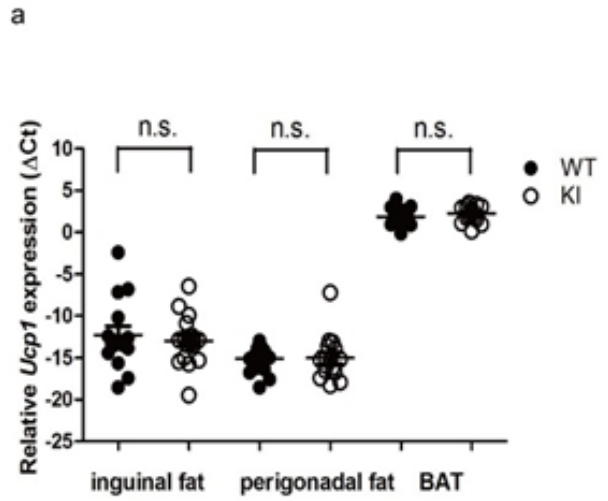

b

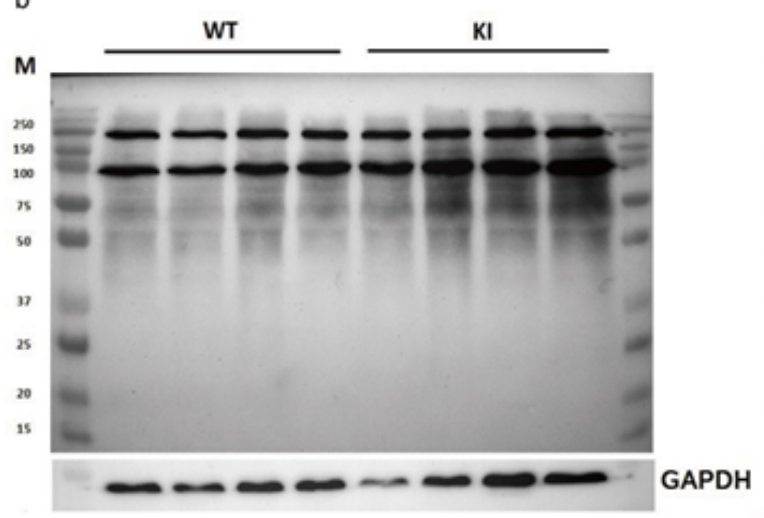

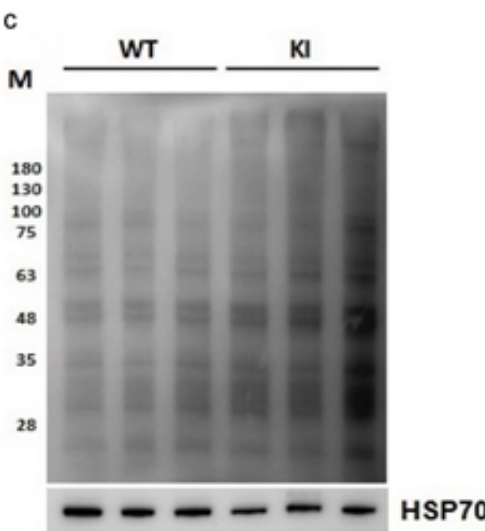

4-HNE-adducted proteins

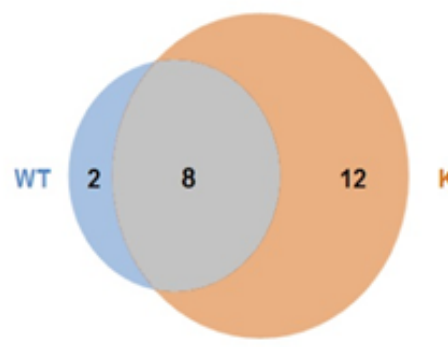

f

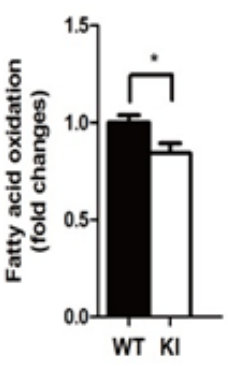

$\mathrm{g}$

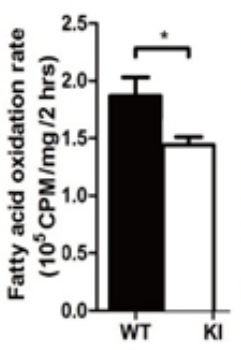

\begin{tabular}{|c|c|c|c|}
\hline $\begin{array}{l}\text { Gene } \\
\text { name }\end{array}$ & protein name & $\begin{array}{l}\text { modified } \\
\text { sites }\end{array}$ & biological function \\
\hline \multicolumn{4}{|c|}{ Aldh2 knock-in mice } \\
\hline Acaa2 & 3-ketoacyl-CoA thiolase, mitochondrial & K25 & Fatty acid beta-oxidation \\
\hline Pcca & Propionyl-CoA carboxylase alpha chain, mitochondrial & K275 & Short-chain fatty acid catabolilsm \\
\hline Ndufc2 & NADH dehydrogenase [ubiquinone] 1 subunit $\mathrm{C} 2$ & $\mathrm{H} 8$ & Mitochondrial ETC complex I \\
\hline Nduffo4 & NADH dehydrogenase [ubiquinone] 1 beta subcomplex subunit 4 & H59 & Mitochondrial ETC complex I \\
\hline Sdhb & Succinate dehydrogenase [ubiquinone] iron-sulfur subunit, milochondrial & H246 & $\begin{array}{l}\text { Mitochondrial ETC complex II, Tricarboxylic } \\
\text { acid cycle }\end{array}$ \\
\hline Potha1 & Pyruvate dehydrogenase E1 component subunit alpha 1 & H121 & Tricarboxylic acid cycle \\
\hline ACO2 & Aconitate hydratase, mitochondrial & K144 & Tricarboxylic acid cycle \\
\hline Hspa8 & Heat shock cognate $71 \mathrm{kDa}$ protein (627aa) & K168 & ATP metabolic process \\
\hline Gpd2 & Glycerol-3-phosphate dehydrogenase & K652 & Glycerol-3-phosphate metabolic process \\
\hline Gpd2 & Glycerol-3-phosphate dehydrogenase & K668 & Glycerol-3-phosphate metabolic process \\
\hline$P C x$ & Pynuate carboxylase & H574 & Pyruvate metabolic process \\
\hline Myh4 4 & Myosin-4 & K1525 & Muscle contraction \\
\hline \multicolumn{4}{|c|}{ Aldh2 knock-in \& wild-type mice } \\
\hline Acaa2 & 3-ketoacyl-CoA thiolase, mitochondrial & K375 & Fatty acid beta-oxidation \\
\hline Uqcrb & Cytochrome b-c1 complex subunit 7 & $\mathrm{H} 39$ & Mitochondrial ETC complex III \\
\hline Uquerc1 & Cytochrome b-c1 complex subunit 1, mitochondrial & $\mathrm{K} 3$ & Mitochondrial ETC complex III \\
\hline Uqurfs1 & Cytochrome b-c1 complex subunit Rieske, mitochondrial & K101 & Mitochondrial ETC complex III \\
\hline Immt & MICOS complex subunit MIC60 & K296 & Maintenance of mitochondrial architecture \\
\hline Immt & MICOS complex subunit MIC60(isoform) & K218 & Maintenance of mitochondrial architecture \\
\hline Atp5f1a & ATP synthase subunit alpha, mitochondrial & K175 & ATP biosynthetic process \\
\hline Hspa8 & Heat shock cognate $71 \mathrm{kDa}$ protein (646aa) & K187 & MAPK signaling pathway \\
\hline \multicolumn{4}{|c|}{ Aldh2 wild-type mice } \\
\hline Chchd3 & MICOS complex subunit & K24 & Maintenance of mitochondrial architecture \\
\hline Atp5a1 & ATP synthase subunit alpha & K125 & ATP synthesis coupled proton transport \\
\hline
\end{tabular}

h
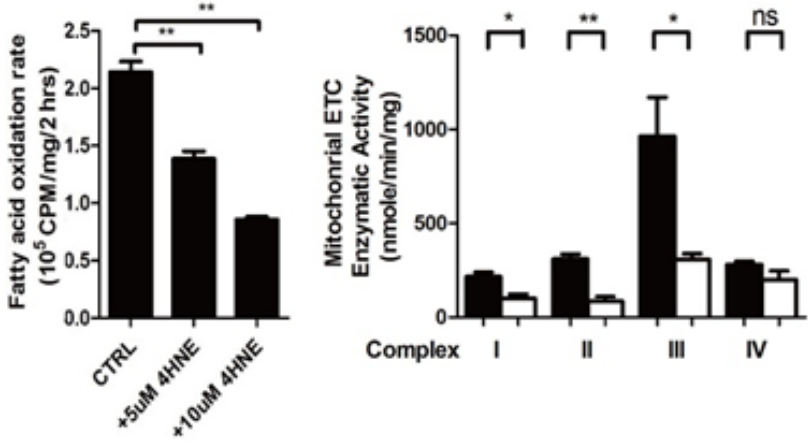

Figure 3

(a) Ucp1 expression levels in inguinal fat, perigonadal fat, and brown adipose tissue (BAT) of the Aldh2 WT and KI mice measured by RT-qPCR ( $\mathrm{n}=21: 17)$. (b) Protein carbonylation of BAT mitochondria by 
hydrazide biotin staining. (c) Immunoblots of 4-hydroxynonenal (4-HNE)-adducted mitochondrial proteins in the BAT from the Aldh2 WT and KI mice on HFHSD. (d,e) 4-HNE-adducted mitochondrial proteins and amino acid residues of the BAT from the Aldh2 KI and WT mice, as identified by LC-MS/MS ( $n=2: 2)$. ( $f$ ) Fatty acid oxidation (FAO) rate of the whole BAT tissue isolated from the Aldh2 WT and KI mice fed a HFHSD ( $n=24: 20)$. (g) FAO of primary brown adipocytes isolated from the Aldh2 WT and KI mice fed a HFHSD ( $n=3$ per group). (h) FAO rate of induced primary brown adipocytes isolated from the WT mice treated with 0,5 , and $10 \mu \mathrm{M} 4-\mathrm{HNE}$ ( $\mathrm{n}=3$ per group). (i) Mitochondrial electron transfer chain (ETC) complex I-IV enzymatic activity isolated from the BAT of Aldh2 WT and KI mice on HFHSD ( $n=4: 4)(j)$ Oxygen consumption rate (OCR) of induced primary brown adipocytes isolated from Aldh2 WT and KI mice fed on HFHSD. Cells were treated with oligomycin, FCCP, and Rotenone/Antimycin A. OCR measurements were normalized to the protein concentration. ( $n=3$ per group) ${ }^{*} P<0.05,{ }^{\star} * P<0.01$. 
a

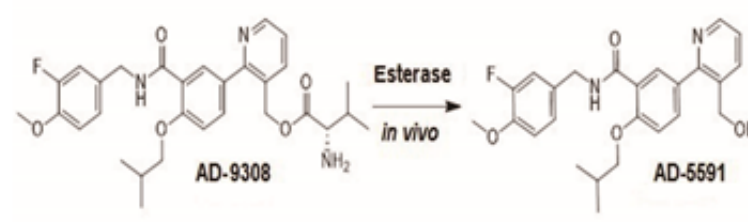

d
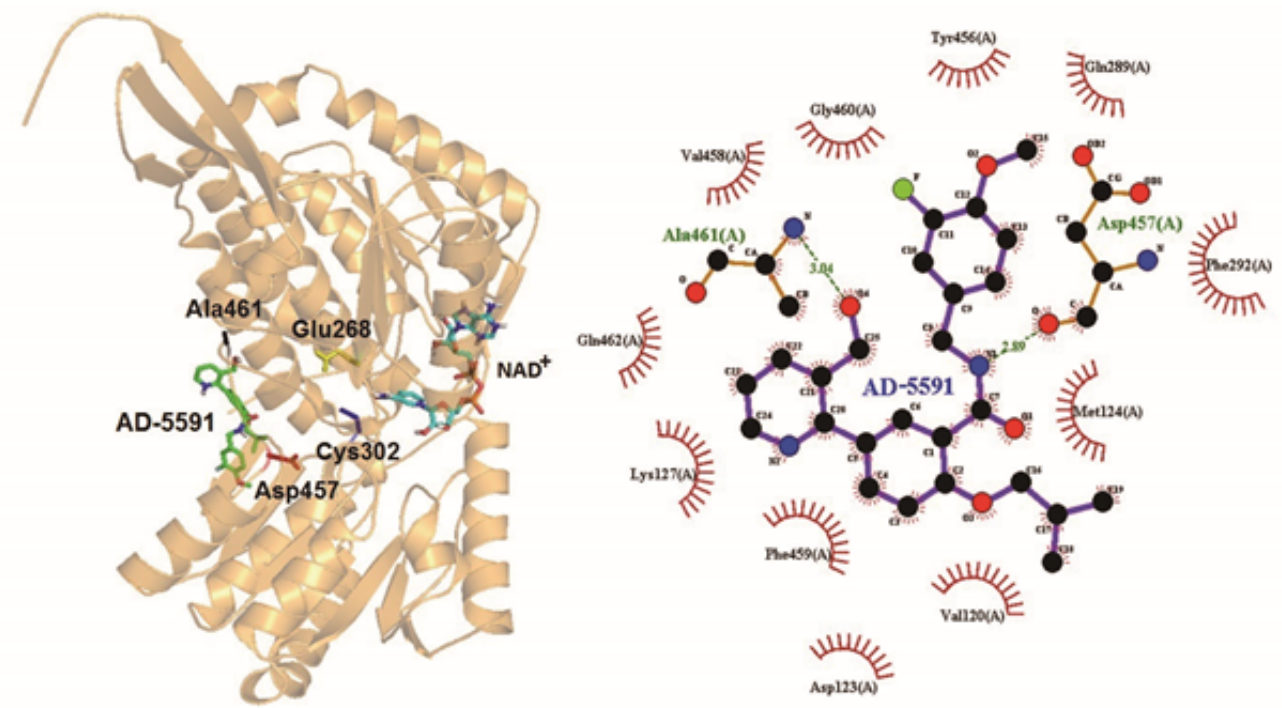

e

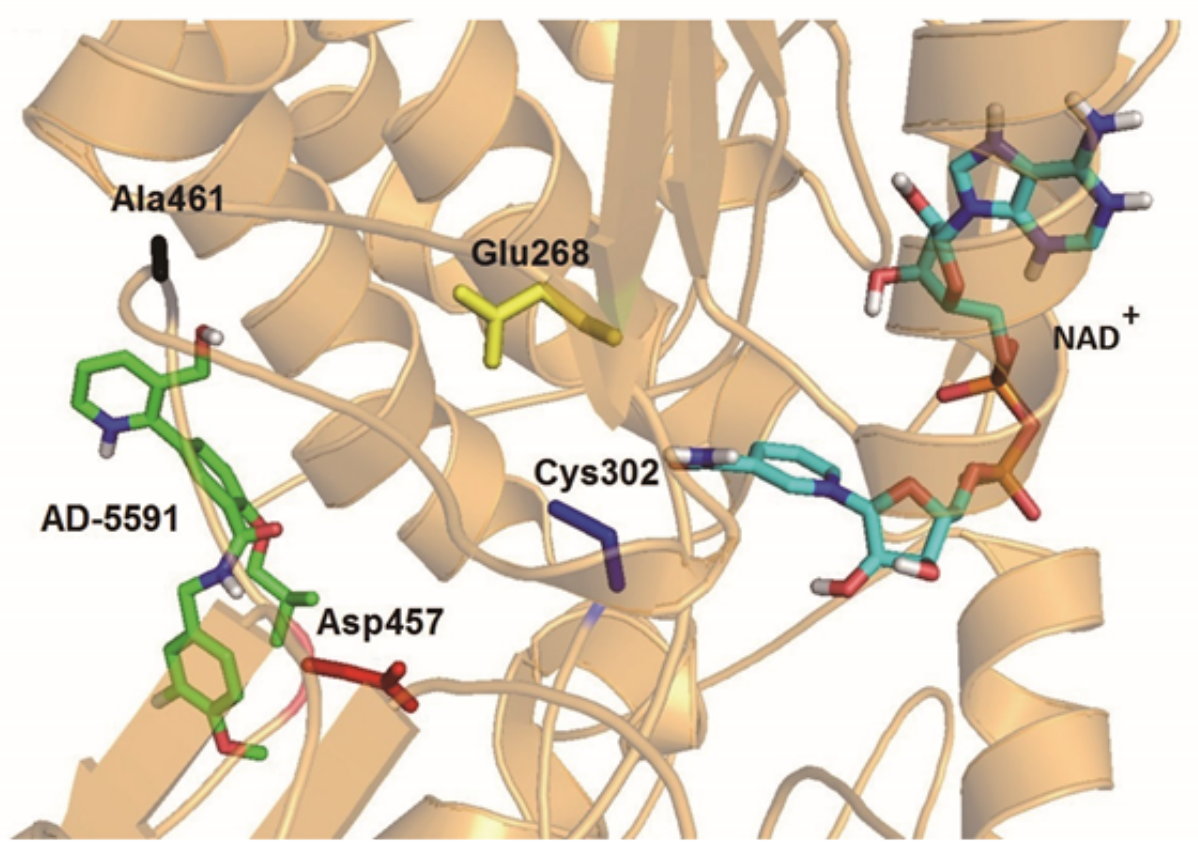

Figure 4

(a) Prodrug AD-9308 is converted to AD-5591 by esterase hydrolysis in vivo. (b) AD-5591 significantly increases the enzymatic activity of recombinant WT and mutant human ALDH2 proteins ( $n=9: 9)$. (c) Ribbon diagram showing the binding pocket of AD-5591 within human WT ALDH2. (d) LigPlot showing the bonds between AD-5591 and human WT ALDH2. (e) Regional ribbon diagram showing the binding of AD-5591 and NAD+ with WT ALDH2. 
a

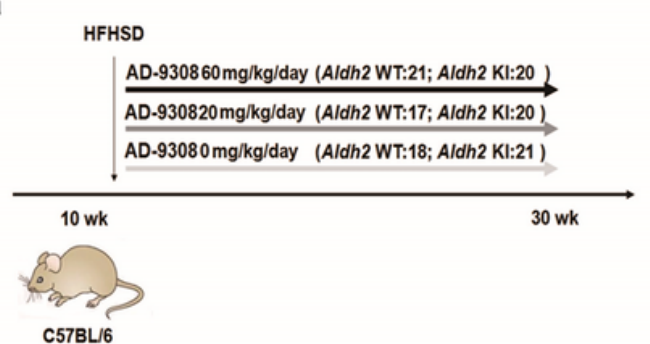

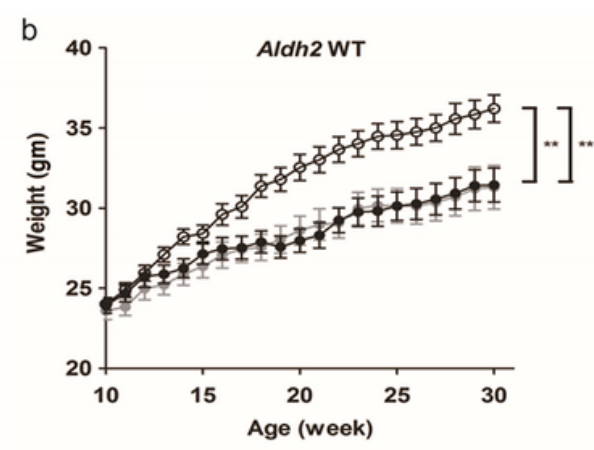
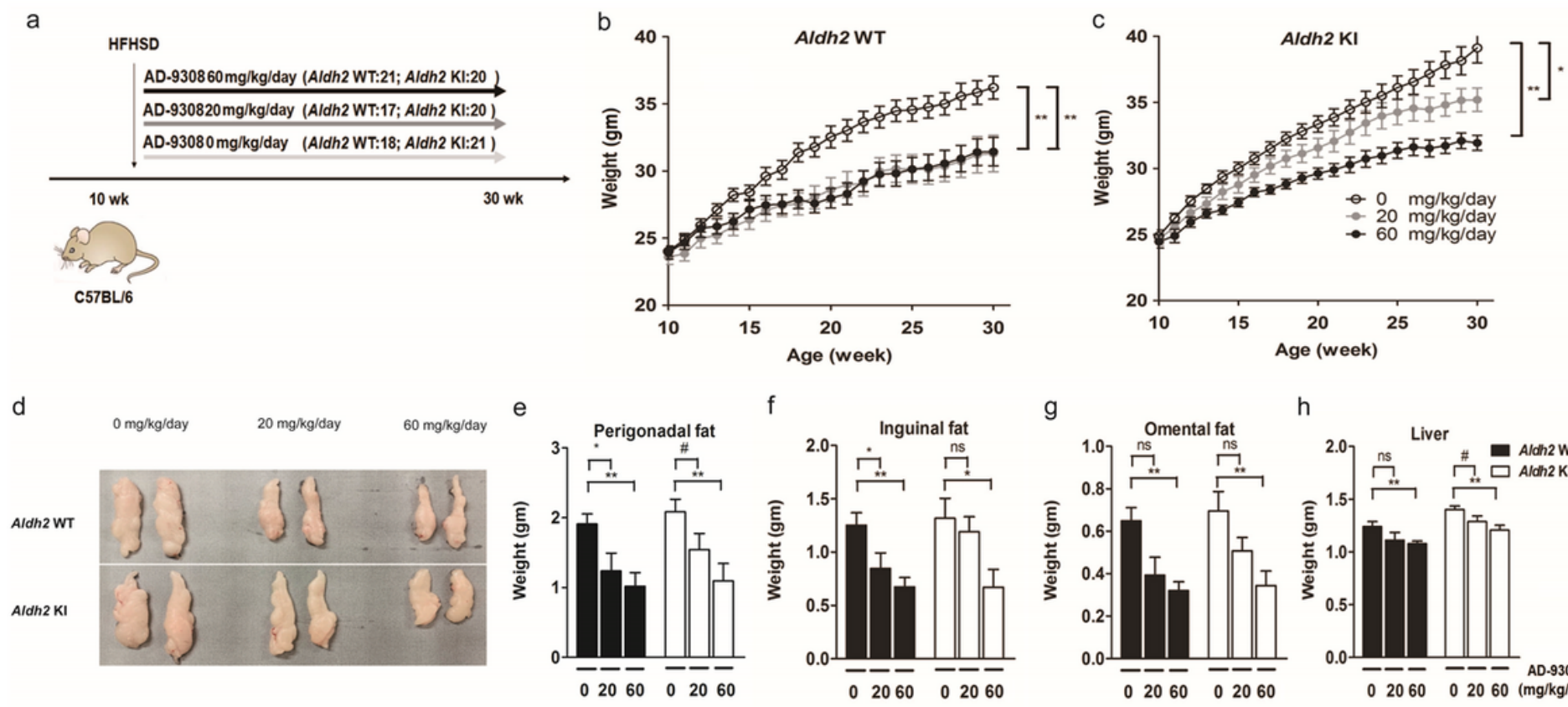

f
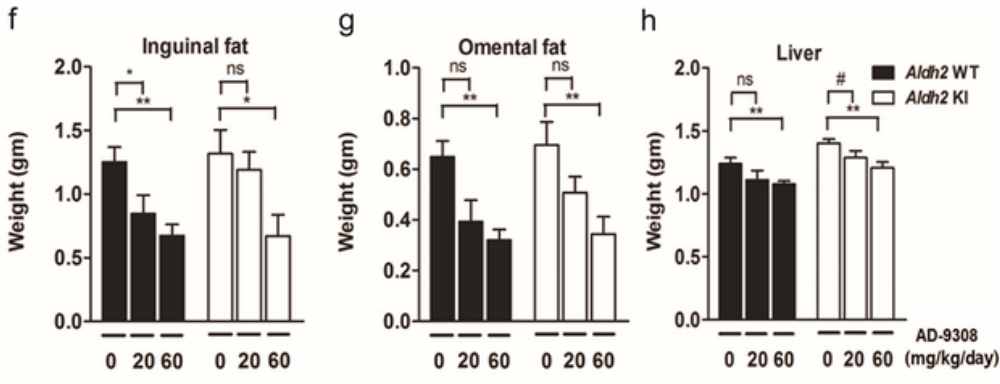

$\mathrm{k}$

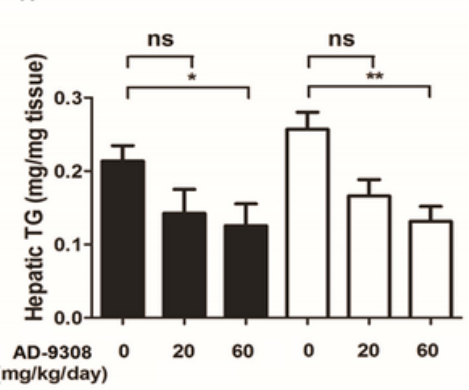

\section{Figure 5}

(a) Study flow of AD-9308 treatment. Body weight of (b) Aldh2 WT and (c) KI mice treated with vehicle, low-dose AD-9308 (20 mg/kg/day), and high-dose AD-9308 (60 mg/kg/day). (d) Gross appearance of the perigonadal fat from the Aldh2 WT and KI mice treated with AD-9308 on HFHSD Weight of (e) perigonadal fat, (f) inguinal fat, $(\mathrm{g})$ omental fat, (h) liver of the Aldh2WT and KI mice treated with AD9308. (i) Gross appearance of the liver. (j) H\&E stained liver, and (k) hepatic triglycerides content of the Aldh2 WT and KI mice treated with AD-9308 on HFHSD ( $n=11: 14$ for the vehicle group; $n=12: 15$ for 20 $\mathrm{mg} / \mathrm{kg} /$ day group; $\mathrm{n}=9: 12$ for $60 \mathrm{mg} / \mathrm{kg} /$ day group). ${ }^{*} \mathrm{P}<0.05 * * \mathrm{P}<0.01$. 
a

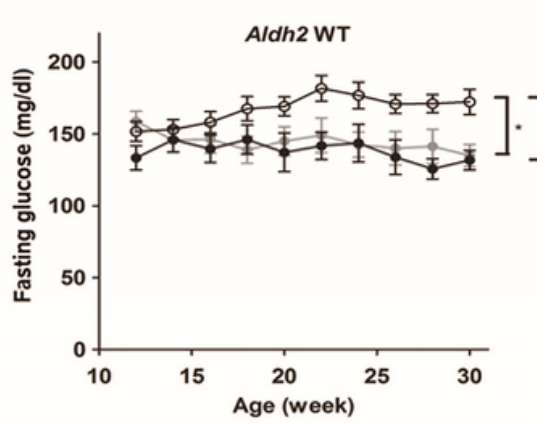

C

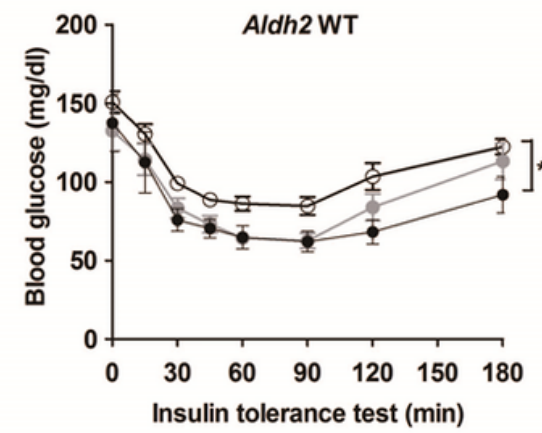

e

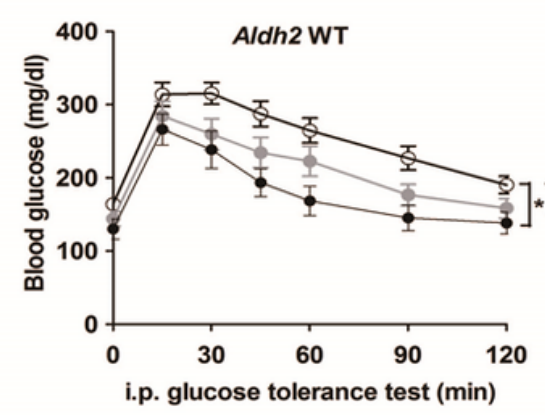

b

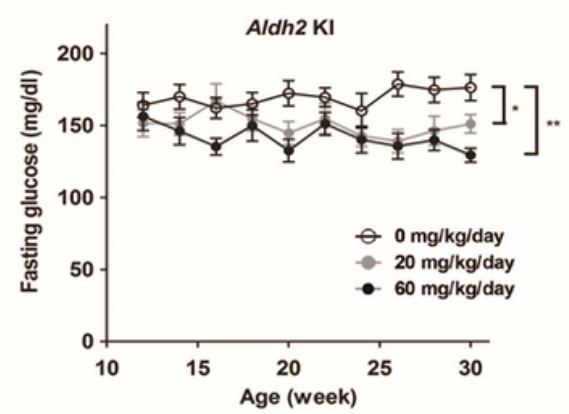

d

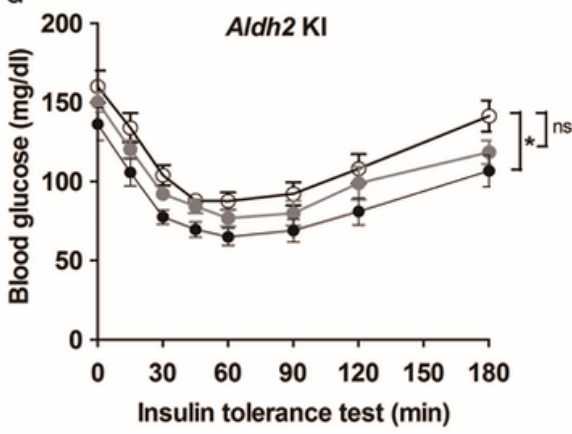

f

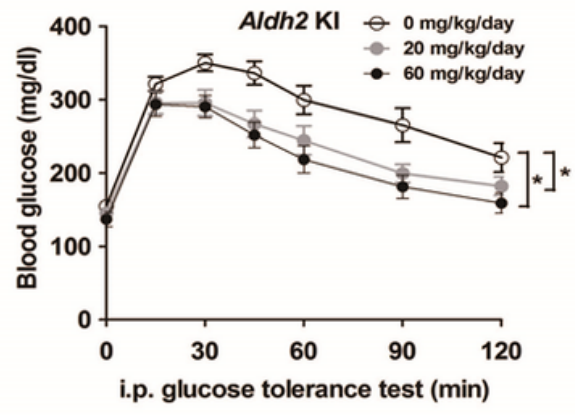

g

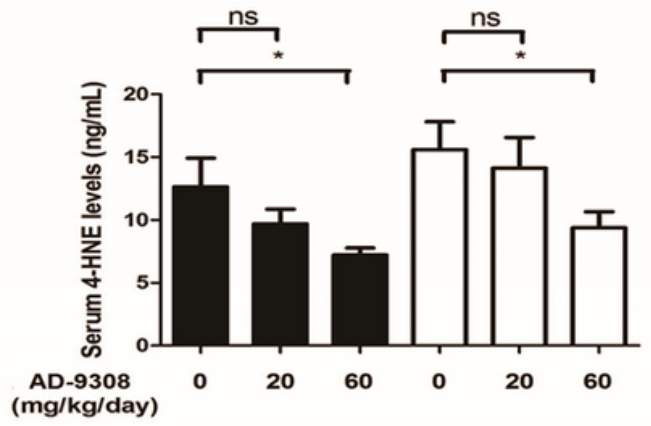

h

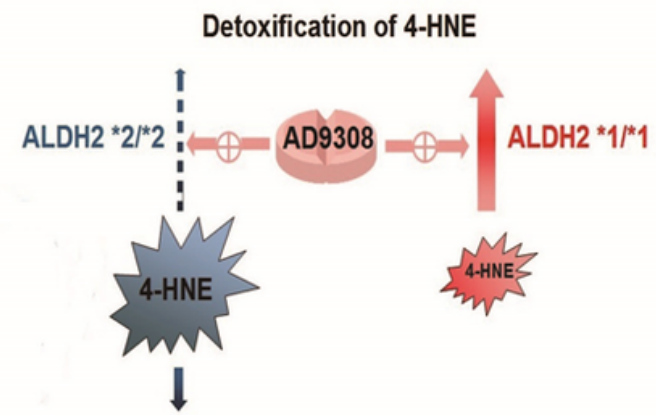

Fatty acid $\beta$-oxidation Electron transport Thermogenesis

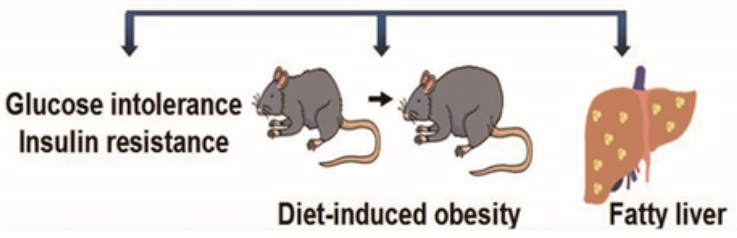

Figure 6

Fasting glucose level of the (a) Aldh2 WT and (b) KI mice treated with vehicle, low-dose AD-9308 (20 $\mathrm{mg} / \mathrm{kg} /$ day), and high-dose AD-9308 (60 mg/kg/day) on HFHSD. (c)(d)Glycemic levels during the insulin sensitivity test and (e)(f) intraperitoneal glucose tolerance test of the Aldh2 WT and KI mice treated with AD-9308 ( $n=15: 15$ for the vehicle group; $n=14: 16$ for $20 \mathrm{mg} / \mathrm{kg} /$ day group; $n=9: 14$ for $60 \mathrm{mg} / \mathrm{kg} /$ day group). (g) Serum 4-HNE levels of the Aldh2 WT and KI mice treated with AD-9308 ( $n=15: 16$ for the vehicle group; $\mathrm{n}=12: 14$ for $20 \mathrm{mg} / \mathrm{kg} /$ day group; $\mathrm{n}=12: 14$ for $60 \mathrm{mg} / \mathrm{kg} /$ day group) on HFHSD. (h) Summary diagram depicting how reducing 4-HNE by AD-9308 ameliorates metabolic disturbances. *P < $0.05 * * P<0.01$.

\section{Supplementary Files}

This is a list of supplementary files associated with this preprint. Click to download. 